\title{
The sub-arcsecond dusty environment of Eta Carinae ${ }^{\star}$
}

\author{
O. Chesneau ${ }^{1}$, M. Min ${ }^{2}$, T. Herbst ${ }^{1}$, L. B. F. M. Waters ${ }^{2}$, D. J. Hillier ${ }^{3}$, Ch. Leinert ${ }^{1}$, \\ A. de Koter ${ }^{2}$, I. Pascucci ${ }^{1}$, W. Jaffe ${ }^{4}$, R. Köhler ${ }^{1}$, C. Alvarez ${ }^{1}$, R. van Boekel ${ }^{2}$, W. Brandner ${ }^{1}$, \\ U. Graser ${ }^{1}$, A. M. Lagrange ${ }^{5}$, R. Lenzen ${ }^{1}$, S. Morel ${ }^{6}$, and M. Schöller ${ }^{6}$ \\ ${ }^{1}$ Max-Planck-Institut für Astronomie, Königstuhl 17, 69117 Heidelberg, Germany \\ e-mail: chesneau@mpia-hd.mpg.de \\ 2 Sterrenkundig Instituut "Anton Pannekoek", Kruislaan 403, 1098 SJ Amsterdam, The Netherlands \\ 3 Department of Physics and Astronomy, University of Pittsburgh, 3941 O'Hara Street, Pittsburgh, PA 15260, USA \\ 4 Leiden Observatory, Niels Bohr weg 2, 2333 CA Leiden, The Netherlands, \\ ${ }^{5}$ Laboratoire d'Astrophysique de l'Observatoire de Grenoble, Université J. Fourier, CNRS, BP 53, 38041 Grenoble Cedex 9 , \\ France \\ ${ }^{6}$ European Southern Observatory, Casilla 19001, Santiago, Chile
}

Received 3 June 2004 / Accepted 8 January 2005

\begin{abstract}
The core of the nebula surrounding Eta Carinae has been observed with the VLT Adaptive Optics system NACO and with the interferometer VLTI/MIDI to constrain spatially and spectrally the warm dusty environment and the central object. In particular, narrow-band images at $3.74 \mu \mathrm{m}$ and $4.05 \mu \mathrm{m}$ reveal the butterfly shaped dusty environment close to the central star with unprecedented spatial resolution. A void whose radius corresponds to the expected sublimation radius has been discovered around the central source. Fringes have been obtained in the Mid-IR which reveal a correlated flux of about $100 \mathrm{Jy}$ situated 0.3 south-east of the photocenter of the nebula at $8.7 \mu \mathrm{m}$, which corresponds with the location of the star as seen in other wavelengths. This correlated flux is partly attributed to the central object, and these observations provide an upper limit for the SED of the central source from $2.2 \mu \mathrm{m}$ to $13.5 \mu \mathrm{m}$. Moreover, we have been able to spectrally disperse the signal from the nebula itself at $\mathrm{PA}=318$ degree, i.e. in the direction of the bipolar nebula $\left(\sim 310^{\circ}\right)$ within the MIDI field of view of $3^{\prime \prime}$. A large amount of corundum $\left(\mathrm{Al}_{2} \mathrm{O}_{3}\right)$ is discovered, peaking at 0. ' $6-1 . \prime 2$ south-east from the star, whereas the dust content of the Weigelt blobs is dominated by silicates. We discuss the mechanisms of dust formation which are closely related to the geometry of this Butterfly nebulae.
\end{abstract}

Key words. techniques: high angular resolution - stars: early-type - stars: winds, outflows - stars: individual: Eta Carinae stars: circumstellar matter

\section{Introduction}

Eta Carinae is one of the best studied but least understood massive stars in our galaxy (Davidson \& Humphreys 1997). With a luminosity of $5 \times 10^{6} L_{\odot}$, it is one of the most luminous stars in the galaxy and at $10 \mu \mathrm{m}$ it is one of the brightest objects outside the solar system (Neugebauer \& Westphal 1969). Eta Car is classified as a Luminous Blue Variable (LBV). Among the most prominent characteristics of the unstable LBV phase are strong stellar winds and possible giant eruptions, which lead to the peeling off of the outer layers of the H-rich stellar envelope and to the formation of small $(\sim 0.2-2 \mathrm{pc})$ circumstellar nebulae (Nota et al. 1995).

During the last two centuries, Eta Carinae has lived through a turbulent history. During the great eruption in the 1840s, the large bipolar nebula surrounding the central object, known as the "Homunculus", was formed. Currently, the Homunculus

* Based on observations collected at the European Southern Observatory, Chile. lobes span a bit less than $20^{\prime \prime}$ on the sky (or $45000 \mathrm{AU}$ at the system distance of $2.3 \mathrm{kpc}$ ) and are largely responsible for the huge infrared luminosity of the system. The cause of the outburst remains unknown. The chemical composition of the Homunculus gas is not known, but some studies of the ionized outer ejecta (Lamers et al. 1998; Smith \& Morse 2004, and references herein) suggest an overabundance of $\mathrm{N}$ and a severe depletion of $\mathrm{C}$ and $\mathrm{O}$. Such an abundance pattern is consistent with CNO equilibrium burning, and suggests a highly evolved star at the base of the "explosion".

The central source has been studied by speckle interferometry techniques, which revealed a complex knotty structure (Weigelt \& Ebersberger 1986; Falcke et al. 1996). Originally, three remarkably compact objects between $0 . ' 1$ and 0.3 northwest of the star were isolated (the so-called BCD Weigelt blobs, the blob A being the star itself). Other similar but fainter objects have since been detected (Weigelt et al. 1995, 1996; Davidson et al. 1997). They are found to be surprisingly bright ejecta moving at low speeds $\left(\sim 50 \mathrm{~km} \mathrm{~s}^{-1}\right)$. They belong to the 
equatorial regions close to the star; their separation from the star is typically $800 \mathrm{AU}$. The large scale equatorial midplane debris disc was nicely revealed with HST data (Morse et al. 1998).

The original detection of a 5.52 year period in Eta Carinae in the spectroscopic and near-infrared photometric data of Damineli (1996) has been confirmed by later observations (Damineli et al. 2000; Davidson et al. 2000; Abraham et al. 2003; Corcoran 2003; Whitelock et al. 2003, 2004). The existence, mass, and orbit of a companion and its possible impact on the behavior of the primary are still strongly disputed (e.g. Davidson 1999; Duncan et al. 1999; Stevens \& Pittard 1999; Corcoran et al. 2001; Feast et al. 2001; Pittard \& Corcoran 2002; Duncan \& White 2003).

Dust plays a key role in the study of Eta Car. It intervenes in every observation as strong and patchy extinction. It also allows the mass of the nebula to be determined. Dust has also been frequently invoked as an important process in explaining the photometric variability of Eta Car. However, the exact nature and location of dust formation/destruction sites has never been observed. Eta Car was observed with the Infrared Space Observatory Observatory (ISO) by Morris et al. (1999). The ISO spectra indicated that a much larger amount of matter should be present around Eta Car in the form of cold dust than previously estimated. Observations with higher spatial resolution by Smith et al. (2002) and Smith et al. (2003a) showed a complex but organized dusty structure within the three inner arcseconds. They showed that the dust content around the star is relatively limited and claimed that the two polar lobes should contain the large mass of relatively cool dust necessary to explain the ISO observations.

The high spatial resolution images of the equatorial regions are puzzling in several ways and raise new key questions: why was the eruption azimuthally asymmetric? Is the complex geometry of the dusty torus a consequence of the 1840 outburst or has it been affected by more recent events? What is the status of the complex Weigelt blob region? Why did the star eject such slow-moving material in its equatorial zone? Does the star form dust continuously, or in episodes related to the mini outburst or the putative wind-wind interaction of Eta Car with its companion?

Improved spatial resolution has enabled the recent progress in our understanding of this emblematic star. HST STIS observations provided the stellar spectrum of Eta Car roughly separated from its nearby ejecta (Hillier et al. 2001). Moreover this impressive instrument has allowed the study of the stellar wind from several points of view at different latitudes in the nebulae by means of P Cygni absorption in Balmer lines reflected in the nebula (Smith et al. 2003b). The authors convincingly prove the asphericity of the wind suggest that the observed enhanced polar wind mass-loss rate may be explained through the theoretical frame developed by Stan Owocki and collaborators (Dwarkadas \& Owocki 2002). In their model, an enhanced mass loss occurs along the rotation axis, due to the large temperature difference between pole and equator, which is in turn caused by the rapid rotation of the star (the von Zeipel effect). Recently, the ionized stellar wind of Eta Carinae has been resolved on the 5 milliarcsecond (mas) scale at a wavelength of $2.2 \mu \mathrm{m}$ with data obtained with VINCI on the Very Large Telescope Interferometer (VLTI, van Boekel et al. 2003). These observations are consistent with the presence of one star which has an ionized, moderately clumpy stellar wind with a mass loss rate of about $1.6 \times 10^{-3} M_{\odot} \mathrm{yr}^{-1}$. This star-plus-wind spherical model, developed by Hillier et al. (2001), is also consistent with the HST STIS observations of the central object. It has also been found that the star is elongated with a de-projected axis ratio of about 1.5 and that the axis itself is aligned with the axis of the large bipolar nebula. These VLTI observations gave an important confirmation of the wind geometry previously proposed by Smith et al. (2003b).

The Hillier model suggests a flux level at $10 \mu \mathrm{m}$ of 200-300 Jy and 10-15 mas diameter of the star plus wind at this wavelength. These dimensions can only be probed using the MIDI instrument at the VLTI, (Leinert et al. 2003a,b). The VINCI observations do not require the presence of other components in the core. In particular, no evidence for a hot dust disc or the putative companion were found. However the presence of warm (300-600 K) dust in the immediate surroundings of Eta Car cannot be excluded since it would be too cool to be detected at $2 \mu \mathrm{m}$.

The MIDI recombiner attached to the VLTI is the only instrument that is able to provide sufficient spatial and spectral resolution in the mid-infrared to disentangle the central components in the Eta Car system from the dusty environment. By definition an interferometer measures a correlated flux, i.e. a flux originating from a source small enough that it is able to produce fringes. The measured correlated flux depends on the source total flux, its geometry and on the length and direction of the projected baseline(s) of the interferometer. We used the $102 \mathrm{~m}$ baseline between the VLT telescopes Antu (UT1) and Melipal (UT3) to observe, for the first time, Eta Car with a resolution of 5-10 mas over the entire $N$ band.

These observations have been complemented with broadand narrow-band observations taken with the NAOS/CONICA (NACO) imager installed on VLT UT4 (Kueyen), equipped with an adaptive optics (AO) system. The diffraction limit of the $8 \mathrm{~m}$ telescope at $3.8 \mu \mathrm{m}$ is about 100 mas. At this wavelength, the NACO adaptive optics is quite sufficient to correct atmospheric seeing, routinely providing a Strehl ratio approaching 0.5. A careful deconvolution procedure can improve the Point Spread Function (PSF) diameter to about 50-80 mas. The NACO observations offer the opportunity to bridge the gap to the MIDI data obtained with very high resolution but sparse UV coverage.

In Sect. 2, we describe the observations and the data reduction. We analyze the NACO and MIDI images in Sect. 3, and we examine the spatial distribution of the dust close to the star in Sect. 4. The information extracted from the correlated flux detected by MIDI is presented in Sect. 5. Finally in Sect. 6, we summarize the implications of the extracted information.

\section{Observations and data reduction}

\subsection{NACO high resolution imaging}

We have observed Eta Carinae with the adaptive optics camera NACO (Lenzen et al. 1998; Rousset et al. 2003) attached 
Table 1. Journal of observations with NACO/UT4. The phase within the 5.52-year cycle is computed from the ephemeris of Daminelli et al. (2000).

\begin{tabular}{|c|c|c|c|c|}
\hline Star & Filter & Camera & Time & $t_{\exp }$ \\
\hline \multicolumn{5}{|c|}{$15 / 16-11-2002, J D=2452625, \Phi=0.87$} \\
\hline$\eta \mathrm{Car}$ & $J$ & S13 & T08:12:00 & $86 \mathrm{~s}$ \\
\hline$\eta \mathrm{Car}$ & $H$ & $\mathrm{~S} 13$ & T08:16:02 & $86 s$ \\
\hline$\eta$ Car & $K \mathrm{~s}$ & S13 & T08:04:21 & $86 s$ \\
\hline$\eta \mathrm{Car}$ & $L^{\prime}$ & L27 & T08:20:24 & $50 \mathrm{~s}$ \\
\hline \multicolumn{5}{|c|}{$16 / 17-11-2002, J D=2452626, \Phi=0.87$} \\
\hline$\eta \mathrm{Car}$ & NB_374 & L27 & T08:47:00 & $50 \mathrm{~s}$ \\
\hline$\eta \mathrm{Car}$ & NB_405 & L27 & T08:50:44 & $50 \mathrm{~s}$ \\
\hline HD 101104 & NB_374 & L27 & T09:10:17 & $50 \mathrm{~s}$ \\
\hline HD 101104 & NB_405 & L27 & T09:17:51 & $50 \mathrm{~s}$ \\
\hline
\end{tabular}

to the fourth $8.2 \mathrm{~m}$ Unit Telescope of the Very Large Telescope (VLT) of the European Southern Observatory (ESO), located at Cerro Paranal, Chile. NAOS was operated in the visual wavefront sensor configuration with the SBRC Aladdin $1024 \times$ 1024 detector. We observed with $J, H, K$ s with the S13 camera and $L^{\prime}$ broad-band filters and the NB_374, NB_405 narrow band filters that cover the emission lines Pfund $\gamma$ and Bracket $\alpha$ respectively with the L27 camera. In camera modes S13 and L27, the fields of view were $14^{\prime \prime} \times 14^{\prime \prime}$ and $28^{\prime \prime} \times 28^{\prime \prime}$ respectively and the pixel scales were 13.25 and 27.1 mas per pixel, sizes sufficient to satisfy the Nyquist sampling criterion. 13.25 mas and 27.1 mas correspond to 30 and $62 \mathrm{AU}$ respectively at the distance of $2.3 \mathrm{kpc}$. The AutoJitter mode was used; that is, at each exposure, the telescope moves according to a random pattern in a $10^{\prime \prime}$ box. Cross-correlation was used to recenter the images at about 0.15 pixel accuracy.

A neutral density filter with an attenuation factor of 70 was necessary in order to avoid saturating the central peak of the PSF. However, the $L^{\prime}$ image was saturated within the inner 0.5 even with shortest exposure time possible $(0.17 \mathrm{~s})$. The NB_405 narrow-band image is not saturated, but the peak intensity of the central source is in the non-linear regime of the detector. The NB_374 narrow-band image does not suffer from this effect by virtue of the lower continuum and line fluxes at this wavelength and the slightly narrower filter.

Individual dithered exposures were co-added, resulting in the total exposure times $t_{\exp }$ shown in Table 1 . The data reduction has been performed using a self-developed IDL routine that processes the individual frames as follows: first, bad pixels are removed. Then, the sky is computed as the mean of the dithered exposures, and subtracted frame by frame. Finally, all the sky-subtracted frames are shifted and added together. The reduced broad-band images are shown in Fig. 1. These broad-band images have not been photometrically calibrated. In Fig. 2, we show a color composite image of the filters $L^{\prime}$, $\operatorname{Br} \alpha$ and $\operatorname{Pf} \gamma$.

The narrow-band images were deconvolved using the Richardson-Lucy algorithm (1974) using as PSF the star HD 101104 observed immediately after the source acquisition. The seeing during the $1 \mathrm{~h}$ narrow-band images observations was stable, typically 0.5 arcsec and the measured FWHM of the PSFs at 3.74 and $4.05 \mu \mathrm{m}$ are 97 and 107 mas respectively, i.e. very close to the diffraction limit of the telescope. By contrast, the $F W H M$ of the central object in Eta Car images in $J$, $H$ and $K$ s are 65, 74 and 77 mas respectively, to be compared with the diffraction limits of 33, 43 and 57 mas. We applied only 40 iterations to enhance the spatial resolution and contrast of the images, stopping before the appearance of any severe artifacts. The resulting Pf $\gamma$ image is shown in Fig. 3. The quality of the deconvolution process can be judged by the comparison of the raw images and the deconvolved ones at iterations 10 and 40 in Fig. 5.

The deconvolved images in the two filters are very similar, apart from the larger extension of the central object at $4.05 \mu \mathrm{m}$. This is obviously an artefact of the deconvolution due to the fact that in the $\operatorname{Br} \alpha$ filter, the 4-6 brightest pixels have entered the non-linear regime of the detector. Therefore, the central object differs from the true telescope PSF referenced with the observation of HD 101104. The distortion of the central peak mimics the flux emitted from a resolved object with the central object appearing larger in the $\operatorname{Br} \alpha$ filter than in the $\mathrm{Pf} \gamma$ one (where the FWHM of the peak is about 60 mas, i.e. $60 \%$ of the diffraction limit). This effect is localized and does not affect the rest of the deconvolved image. Indeed one can check in Fig. 5 that all the structures are in common between both filters.

We attempted to flux-calibrate the NB_374 and NB_405 images by using the AO calibrator, HD 101104 observed immediately after Eta Car. HD 101104 is a M4III star (Dumm \& Schild 1998) which has been chosen for brightness considerations and not with the purpose of photometric calibration. Hence this target is not well suited for such a task but we attempted anyway to calibrate the flux received by the narrow-band filters. From the typical intrinsic color $K-L^{\prime}=0.21$ of an M4III star, and the measured stellar $K$-band magnitude of $m_{K}=0.0 \pm 0.1$, its $L^{\prime}$ magnitude is estimated to be $m_{L^{\prime}}=-0.2 \pm 0.1$. Within the $L^{\prime}$ filter, the $3.74 \mu \mathrm{m}$ region of a M4 star is relatively free from lines, but the $4.05 \mu \mathrm{m}$ region is strongly affected (Fluks et al. 1994). Therefore, the intrinsic color of HD 101104 within the NB_374 filter is about $\mathrm{K}-\mathrm{NB} \_374=0.15 \pm 0.1$, which means that the magnitude of HD 101104 within this filter is $m_{3.74}=-0.15 \pm 0.1$.

We estimated the $L^{\prime}$ magnitude of Eta Car to be $L^{\prime}=$ $-1.85 \pm 0.2$ within a circle of $3^{\prime \prime}$, based on the flux received in the $L^{\prime}$ filters in the non-saturated regions and also from the flux received in the $3.74 \mu \mathrm{m}$ filter. The evolution of the magnitude with the encircled flux is shown in Fig. 4. A non-negligible flux is of course emitted outside the studied regions but the dynamic range reached with the NACO short exposures is too limited to allow a good photometric estimation outside this radius and the photometry computed here is probably underestimated. The quasi-simultaneous $L$ band magnitudes (centered at $3.45 \mu \mathrm{m}$ and not at $3.8 \mu \mathrm{m}$ ) from Whitelock et al. 2004 are -1.737 for JD $=2452603.60$ (November 25, 2002) and -1.761 for JD $=2452661.57$ (January 22, 2003). By scaling the PSF flux to the flux of the central source we estimate the stellar contribution in the NB_374 filter to be $520 \pm 70 \mathrm{Jy}$ or $m_{3.74}=-0.8 \pm 0.3$. 

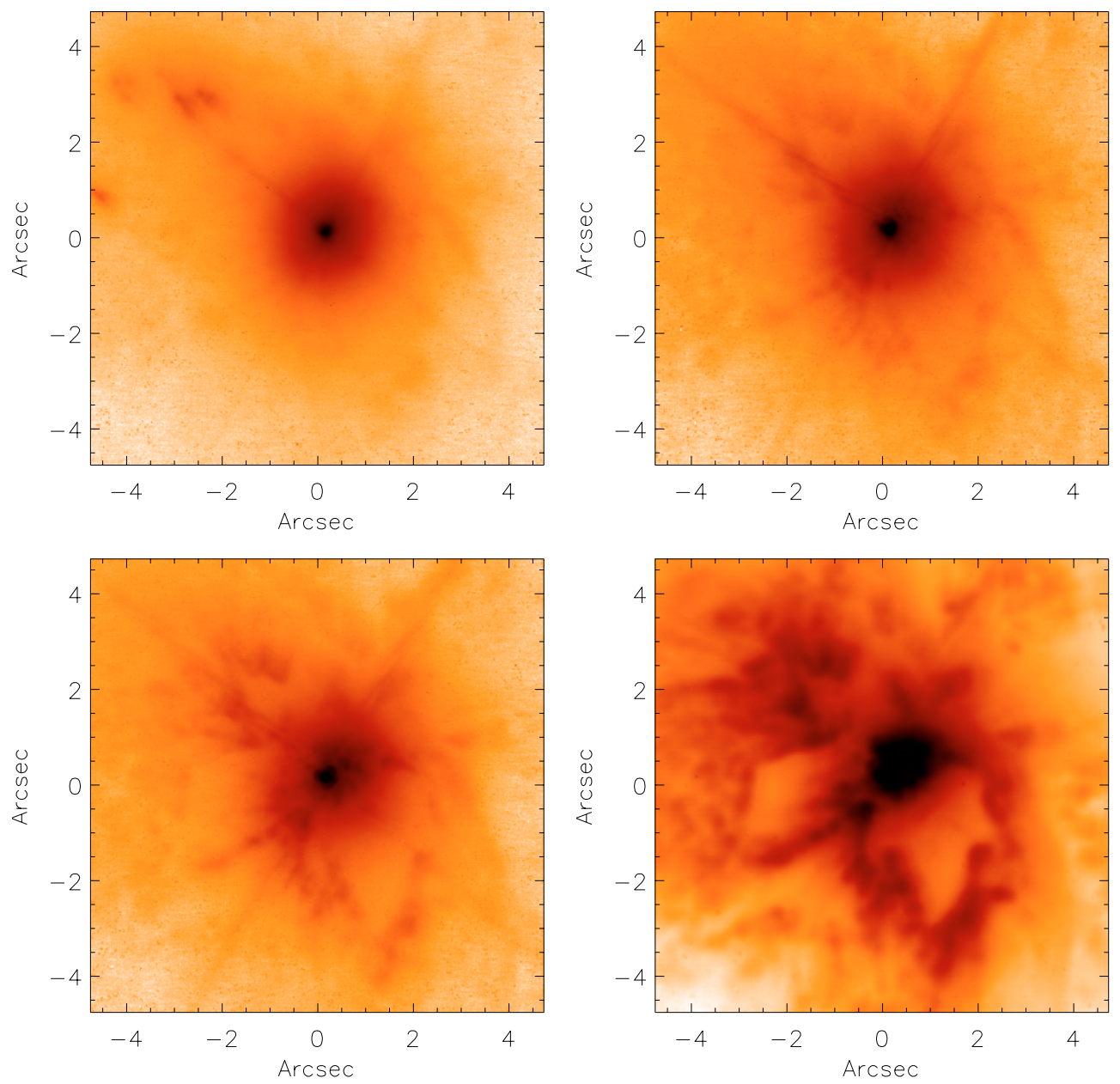

Fig. 1. From left to right and up to down, $J, H, K \mathrm{~s}, L^{\prime}$ images from NACO shown in logarithmic inverted scale. The $L^{\prime}$ image is slightly overexposed in spite of the smallest possible integration time.

\subsection{MIDI observations}

\subsubsection{Observing sequence}

Eta Car was also observed with MIDI (Leinert et al. 2003a,b) the mid-infrared recombiner of the Very Large Telescope (VLT). The VLTI/MIDI interferometer operates like a classical Michelson interferometer to combine the MIR light ( $N$ band, 7.5-14 $\mu \mathrm{m}$ ) from two VLT Unit Telescopes (UTs). For the observations presented here, the UT1 and the UT3 telescopes were used, separated by $102 \mathrm{~m}$ with the baseline oriented $56^{\circ}$ $(\mathrm{E}$ of $\mathrm{N})$.

The observing sequence, typical of interferometric measurements, is influenced by the design of the instrument (Leinert et al. 2003a,b; Przygodda et al. 2003). The chopping mode ( $f=2 \mathrm{~Hz}$, angle -90 degree) is used to visualize and accurately point at the star. The detector pixel size projected on the sky is 98 mas (measured by observations of close visual binaries) and the field of view (FOV) is limited to $3^{\prime \prime}$. The number of frames recorded for each image was generally 2000, and the exposure time per frame is $4 \mathrm{~ms}$ to avoid fast background saturation. If the result of the centering is not satisfactory, the procedure is started again.
Then, the MIDI beam combiner, the wide slit $\left(0 .{ }^{\prime} 6 \times 3^{\prime \prime}\right)$, and the $\mathrm{NaCl}$ prism are inserted to disperse the light and search for the fringes by moving the VLTI delay lines. The resulting spectra have a resolution $\lambda / \Delta \lambda \sim 30$. When searching for the fringe signal, the large delay line of the VLTI is moved to compensate for Earth rotation and atmospheric delays, while the MIDI internal piezo-driven delay line is driven in scans to create the fringe pattern. Once the fringes are found a file is recorded while MIDI tracks them. Finally, two other files are recorded for the photometry. In the first file, one shutter only is opened, corresponding to the calibration of the flux from UT1 and the flux is then divided by the MIDI beam splitter and falls on two different regions of the detector. The total flux is determined separately by chopping between the object and an empty region of the sky, and determining the source flux by subtraction. Then the same procedure is applied with UT3.

A list of all observations is presented in Table 2. The scientific observations have been mainly conducted in the night of the 16th and 17th of June 2003. Unfortunately, Eta Car was only observable at the very beginning of the night and has consequently been observed with two close sky projected baselines of $74 \mathrm{~m}$ and $78 \mathrm{~m}$ and $\mathrm{PA}=57^{\circ}$ and $62^{\circ}$ respectively. We also report some observations which were performed 


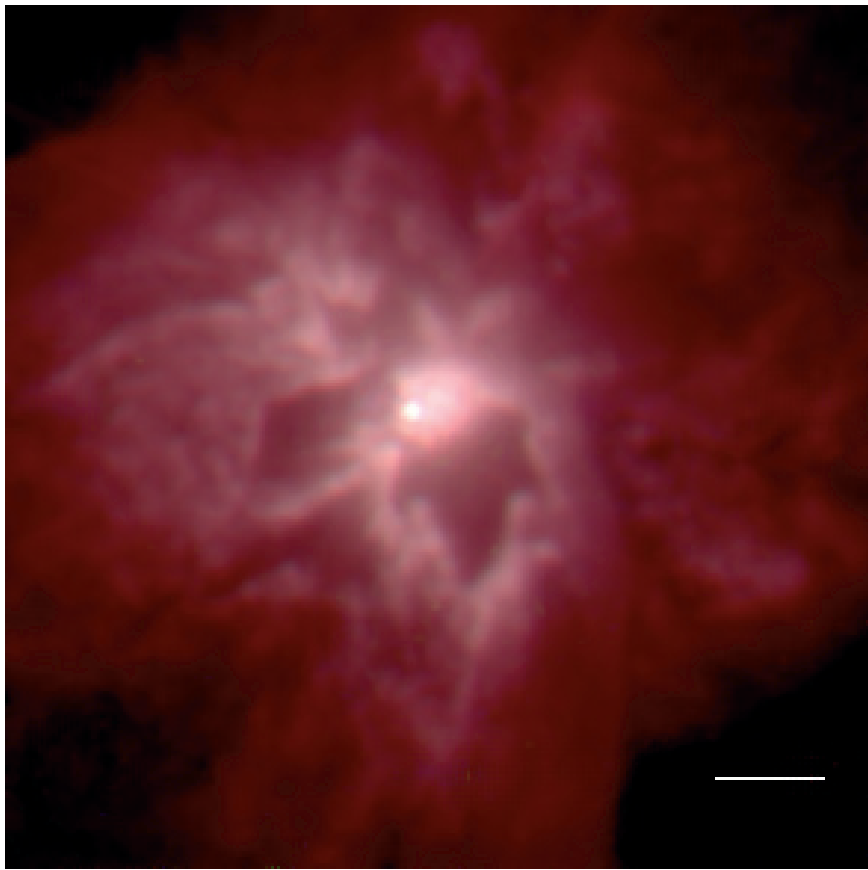

Fig. 2. Color image combining the flux from $L^{\prime}$ (red) and $\operatorname{Br} \alpha$ (green) and Pf $\gamma$ (blue) filters. At these wavelengths, the hydrogen emission represents only a small amount of the flux: about $10 \%$ and $2 \%$ of flux recorded with the $\operatorname{Br} \alpha$ and $\operatorname{Pf} \gamma$ filter respectively. The $L^{\prime}$ filter shows deeper details of the nebula but is saturated in the $0 .{ }^{\prime} 5 \times 0 .{ }^{\prime} 5$ core. The white bar represents $1^{\prime \prime}$.

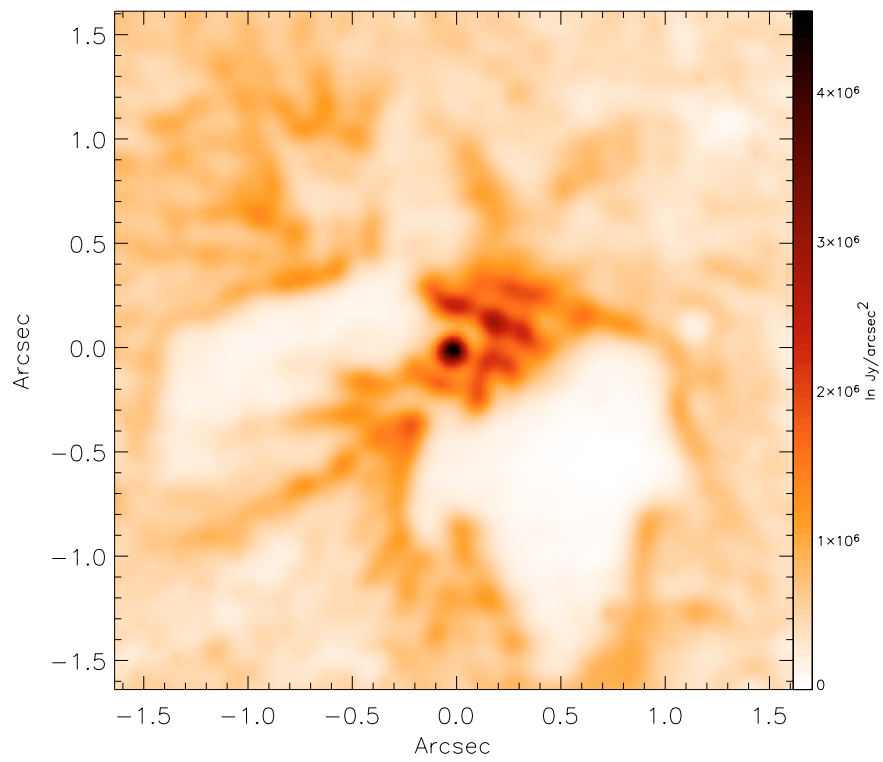

Fig. 3. Pf $\gamma$ deconvolved image. The resolution achieved is in the order of 60 mas. To enhance the contrast, the image $I^{1 / 4}$ is shown. The color scale is expressed in Jy/arcsec ${ }^{2}$. Taken into account the large error bars of the photometry, this scale is only indicative of the flux.

in February 2003 during the first MIDI commissioning run (see Sect. 5.1). These observations were carried out when the MIDI fringe tracker was not performing well and the sensitivity is quite limited compared to the measurements performed

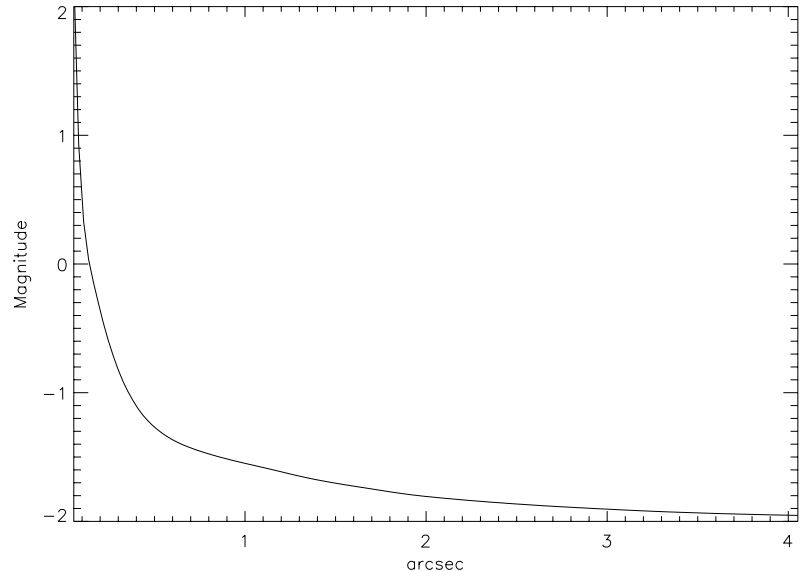

Fig. 4. Evolution of the $L^{\prime}$ magnitude based on aperture photometry with increasing radius. The integrated flux within a circle of $3^{\prime \prime}$ is $L^{\prime}=-1.85 \pm 0.2$.

in June. However, some data recorded in undispersed mode are of particular interest and are presented here (Sect. 5.1).

\subsubsection{Acquisition images}

Custom software, written in the IDL language was used to reduce the images, spectra and fringe data. MIDI is a relatively new and unique instrument. The MIDI data reduction is described more extensively in Leinert et al. (2004) and a devoted paper is in preparation.

The first step of the reduction is to read in the acquisition datasets, average the frames on the target and the frames on the sky, and subtract the average sky frame from the average target frame. Despite the high number of optical elements in the VLTI/MIDI system (33 in total), the quality of the $8.7 \mu \mathrm{m}$ images is comparable to the best Mid-IR images published to date (Smith et al. 2002). The spatial resolution has been slightly increased by performing a deconvolution using 40 iterations of the Lucy-Richardson algorithm and the result is shown in Fig. 9. The spatial resolution reached after the treatment is about 150 mas. Most MIDI targets are unresolved by a single $8 \mathrm{~m}$ telescope; thus many PSF samples are available for testing the quality of the deconvolution process. We used as PSF reference the acquisition images of HD 120323 (2 Cen, M4.5III, $\left.m_{8.7}=-1.8\right)$, HD $148478\left(\alpha\right.$ Sco, M1.5Ib, $\left.m_{8.7}=-4.34\right)$ and HD 151680 ( $\epsilon$ Sco, K2.5III, $m_{8.7}=-0.37$ extrapolated $)$.

The $8.7 \mu \mathrm{m}$ magnitudes have been extracted from the Catalog of Infrared Observations (Edition 5, Gezari+ 1999). Accurate $8.7 \mu \mathrm{m}$ photometry of the MIDI acquisition images is difficult. First, the acquisitions from Eta Car and $\alpha$ Sco were slightly saturated, which affects the linearity of the detector response but also its local offset. Then, due to a pupil mismatch, the FOV and also the background level are different between the two telescopes. For UT3, the FOV was about $3^{\prime \prime} \times 2^{\prime \prime}$ while for UT1 the FOV was less than $2^{\prime \prime} \times 2^{\prime \prime}$. We end up with an integrated $8.7 \mu \mathrm{m}$ magnitude within UT3 and UT1 FOVs of $-5.65 \pm 0.3$ and $-5.45 \pm 0.3$ respectively. One of the MIDI acquisition images from UT3 is shown in Fig. 10 and the best deconvolved $8.7 \mu \mathrm{m}$ image is shown in Fig. 9. The flux scale 

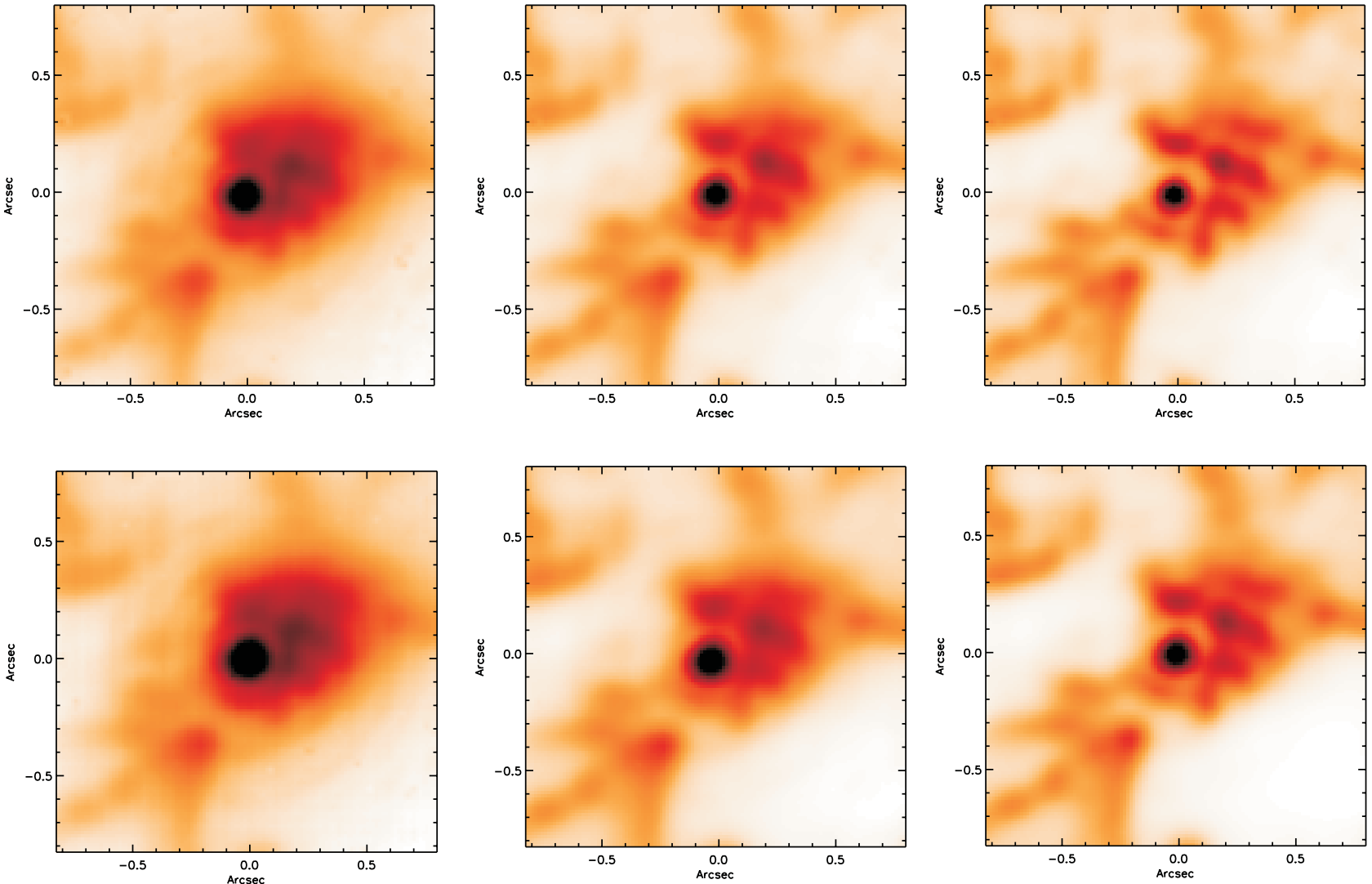

Fig. 5. Zoom into the deconvolved images from the NB_3.74 (up) and NB_4.05 (bottom) filters. The raw images are shown in the left side, the deconvolved images at iteration 10 and 40 are shown in the middle and the in the right side. The resulted images from the two filters are fairly similar except for the size of the central source which is $25 \%$ larger in $\mathrm{Br} \alpha$ due to a slight non-linearity of the detector at high flux regime.

given in Fig. 9 should be considered only as an indication due to the large errors mentioned above and also due to the further difficulties in the deconvolution process.

\subsubsection{Dispersed photometry}

The second reduction step consists of reading the dispersed photometric datasets used for the calibration of the contrast of the dispersed fringes. We use the same procedure to average the frames on the target and the frames on the sky, and then subtract the average sky frame from the average target frame. Eta Car is a complex extended object which requires a dedicated reduction procedure. In the following we describe the "classical" data reduction of MIDI data applied for sources unresolved by a single dish telescope, i.e. for the calibrators.

In the averaged, subtracted frame, the wavelength axis is oriented along the horizontal detector axis. For each detector column, the vertical centroid and width of the spectrum are estimated by fitting a Gaussian function to the peak. The centroid position in all illuminated columns is fitted with a quadratic polynomial as a function of column number, while the width is fitted by a linear function. This procedure is carried out on both photometric datasets (corresponding to telescope UT1 and UT3 respectively). Both fits are averaged and used to create a 2-dimensional weighting mask consisting of a Gaussian function with the average position and width of the spectra for each column. This mask is applied to both photometric and interferometric data to supress noise from regions where few source photons fall (Sect. 2.2.5).

We used HD 167618 ( $~ \eta$ Sgr $)$ and HD 168454 ( $\delta$ Sgr $)$ as calibrators for the dispersed photometry. They are secondary flux calibrators which were observed several times during the June run and have been calibrated using two primary calibrators ( $\alpha$ Aql and HD 177716) for which very good quality spectra are available (Cohen et al. 1998). The airmass correction is extracted from the observations of HD 168454 at two different airmasses.

Considering the very good atmospheric conditions encountered during this observing run, the $N$-band images are close to the diffraction limit. This implies that we can obtain about 8-10 independent spectra of the nebula with a mean spatial $F W H M$ of about 250 mas. This information is valuable for the study of the nebula dust content and provide complementary information to the correlated flux study described in Sect. 5.

A set of spatially resolved spectra of Eta Car were extracted along the slit direction using a modified version of the MIDI photometric algorithm. A "point source" weighting mask was constructed from a calibrator, and then shifted along the slit to extract a sequence of spectra from Eta Car itself. The stability of MIDI within its cryostat permits such a procedure. The purpose is to avoid a bias on the trace due to the shift of the emission photocenter with wavelength in the $N$ band towards 
Table 2. Journal of observations with MIDI/UT1-UT3. The phase within the 5.52-year cycle is computed from the ephemeris of Daminelli et al. (2000).

\begin{tabular}{|c|c|c|c|}
\hline 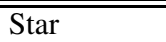 & Template & $\overline{\text { Time }}$ & Frames \\
\hline \multicolumn{4}{|c|}{$23 / 24-02-2003, \mathrm{JD}=2452695, \Phi=0.91, B=75 \mathrm{~m}, \Theta=60$} \\
\hline$\eta$ Car & Search. undispersed & T07:06:22 & 9000 \\
\hline$\eta$ Car & Track. undispersed & T07:09:25 & 9000 \\
\hline$\eta \mathrm{Car}$ & Track. dispersed & T07:15:01 & 9000 \\
\hline$\eta$ Car & Search. undispersed & T07:20:01 & 2800 \\
\hline$\eta$ Car & Search. undispersed & T07:24:03 & 2800 \\
\hline$\eta$ Car & Phot. UT1 disp. & T07:36:42 & 400 \\
\hline$\eta$ Car & Phot. UT3 disp. & T07:38:59 & 400 \\
\hline \multicolumn{4}{|c|}{$12 / 13-06-2003, \mathrm{JD}=2452893, \Phi=0.96, B=74 \mathrm{~m}, \Theta=62^{\circ}$} \\
\hline$\eta$ Car & Track. dispersed & T00:31:24 & 9000 \\
\hline$\eta$ Car & Track. dispersed & T00:35:43 & 10000 \\
\hline$\eta \mathrm{Car}$ & Track. dispersed & T00:39:17 & 7500 \\
\hline$\eta$ Car & Phot. UT1 disp. & T00:43:53 & 3000 \\
\hline$\eta$ Car & Phot. UT3 disp. & T00:45:55 & 3000 \\
\hline \multicolumn{4}{|c|}{$14 / 15-06-2003, \mathrm{JD}=2452806, \Phi=0.96, B=78 \mathrm{~m}, \Theta=56^{\circ}$} \\
\hline HD 120323 & Acquisition $\mathrm{N} 8.7 \mu \mathrm{m}$ & T23:08:03 & 2000 \\
\hline HD 120323 & Acquisition $\mathrm{N} 8.7 \mu \mathrm{m}$ & T23:09:16 & 2000 \\
\hline HD 120323 & Track. dispersed & T23:18:08 & 12000 \\
\hline HD 120323 & Phot. UT1 disp. & $\mathrm{T} 23: 23: 24$ & 3000 \\
\hline HD 120323 & Phot. UT3 disp. & $\mathrm{T} 23: 25: 28$ & 3000 \\
\hline$\eta \mathrm{Car}$ & Acquisition $\mathrm{N} 8.7 \mu \mathrm{m}$ & T23:47:45 & 2000 \\
\hline$\eta$ Car & Acquisition $\mathrm{N} 8.7 \mu \mathrm{m}$ & $\mathrm{T} 23: 48: 51$ & 2000 \\
\hline$\eta$ Car & Track. dispersed & $\mathrm{T} 23: 55: 47$ & 12000 \\
\hline$\eta \mathrm{Car}$ & Phot. UT1 disp. & T00:02:10 & 3000 \\
\hline$\eta$ Car & Phot. UT3 disp. & T00:03:49 & 3000 \\
\hline HD 148478 & Acquisition $\mathrm{N} 8.7 \mu \mathrm{m}$ & T00:14:53 & 2000 \\
\hline HD 148478 & Acquisition $\mathrm{N} 8.7 \mu \mathrm{m}$ & T00:17:01 & 2000 \\
\hline HD 151680 & Acquisition $\mathrm{N} 8.7 \mu \mathrm{m}$ & T00:24:12 & 2000 \\
\hline HD 151680 & Acquisition $\mathrm{N} 8.7 \mu \mathrm{m}$ & T00:25:23 & 2000 \\
\hline HD 151680 & Track. dispersed & T00:32:49 & 12000 \\
\hline HD 151680 & Phot. UT1 disp. & T00:38:22 & 2000 \\
\hline HD 151680 & Phot. UT3 disp. & T00:40:08 & 2000 \\
\hline HD 167618 & Acquisition $\mathrm{N} 8.7 \mu \mathrm{m}$ & T01:18:33 & 2000 \\
\hline HD 167618 & Acquisition $\mathrm{N} 8.7 \mu \mathrm{m}$ & T01:19:41 & 2000 \\
\hline HD 167618 & Track. dispersed & T01:38:28 & 12000 \\
\hline HD 167618 & Phot. UT1 disp. & T01:33:15 & 3000 \\
\hline HD 167618 & Phot. UT3 disp. & T01:35:28 & 3000 \\
\hline HD 168454 & Acquisition $\mathrm{N} 8.7 \mu \mathrm{m}$ & T02:35:55 & 2000 \\
\hline HD 168454 & Acquisition $\mathrm{N} 8.7 \mu \mathrm{m}$ & T02:37:12 & 2000 \\
\hline HD 168454 & Track. dispersed & T02:40:47 & 12000 \\
\hline HD 168454 & Phot. UT1 disp. & T02:50:18 & 3000 \\
\hline HD 168454 & Phot. UT3 disp. & $\mathrm{T} 02: 52: 34$ & 3000 \\
\hline
\end{tabular}

the north-west, due to the increasing contribution of colder dust situated in the Weigelt complex (Smith et al. 2002).

The slit was positioned at $\mathrm{PA}=140^{\circ} \pm 3^{\circ}$, i.e. very close to the nebular principal orientation. Nine spectra separated by 400 mas have been extracted with sufficient SNR, the five central ones using a Gaussian weighting function with a FWHM of 200 mas and the four external ones with a slightly larger beam in order to increase the SNR (which implies a slight cross-talk between the beams). The parameters of the apertures are reported in Table 4 and the spectra are presented in Fig. 11.

\subsubsection{Undispersed correlated flux}

The spatial distribution of the fringes detected by MIDI with the $8.7 \mu \mathrm{m}$ filter is shown in Fig. 12. The data were recorded during February commissioning time when the main observing modes of MIDI had not yet "crystallized". This figure is very interesting because it is one of the first wide field interferometric detections of fringes reported to date. The fringes are detected by measuring the fluctuation of the detector power, pixel-by-pixel, as the OPD is scanned. The level of the detector, background and sky noise are clearly identified and well constrained by the multiple tests performed on the sky during MIDI commissioning. By choosing the frames for which fringes are detected we can localize the fringe signal to an area of about one PSF diameter. Since fringes are detected only in the overlapping part of the beams coming from the individual UTs, the spot visible in calibrators exhibits a sightly smaller $F W H M$ than in the non-interferometric acquisition images. We have checked that the calibrators observed before and after Eta Car have a similar extension by performing a 2D Gaussian fit. Due to the individual pointing errors from each telescope, the overlap spot is not necessarily perfectly symmetric, but no asymmetry larger than $15 \%$ is observable. In contrast to calibrators, the fringes from Eta Car are more extended than the acquisition PSF. To verify the extension of this signal we have first removed a noise pattern extracted at OPD positions farther than $1 \mathrm{~mm}$ away from the white light fringe $(\mathrm{OPD}=0)$, i.e. at a location where no fringes are present. A typical spot extracted from a calibrator was then centered on the maximum, scaled and subtracted from the figure. This technique is similar to the one used to remove a PSF from images.

\subsubsection{Dispersed correlated flux}

Each frame of the fringe data is reduced to a one-dimensional spectrum by multiplying it with the mask, integrating in the direction perpendicular to the spectral dispersion, and finally subtracting the two oppositely phased output channels of the beam combiner from each other. The spectra from each scan with the piezo-mounted mirrors are collected and fourier-transformed with respect to OPD. The fringe amplitude at each wavelength is then obtained from the power spectrum. We typically summed four pixels in the dispersion direction to improve the signal-to-noise ratio.

The correlated flux varies from scan to scan due to the variable overlap of the two telescope beams. The scans used to estimate the flux were selected based on the white-light fringe amplitude, i.e. the fringe amplitude that is seen after integrating over all usable wavelengths. The histogram of all white-light fringe amplitudes within a fringe track dataset usually shows a small peak near zero, and a broad peak at higher amplitudes. We interactively set a threshold just below this broad peak, and average the fringe amplitudes of all scans with a white-light fringe amplitude higher than this threshold to give the final fringe amplitude as a function of wavelength. The raw visibility is obtained by dividing the fringe amplitude by the total photometric flux. The calibrated visibility is obtained by dividing the raw visibility of an object by that of an unresolved 
calibrator. The photometrically calibrated flux creating the fringes is called correlated flux.

The MIDI reduction software has been modified to allow it to handle spatially extended fringes in the slit direction. The extraction mask, which is usually wide in the slit direction to include all the light from the sources has been narrowed to cover no more than 3-4 pixels (i.e. 0.'3-0.'4) along the slit. We used the set of masks created for the photometric study described in Table 4. At first we checked that the calibrated visibilities of calibrators derived with this mask were identical to the ones derived with the normal width. It turned out that the instrumental visibility is slightly higher (about 5-10\%, depending on the wavelength) when the mask is narrower. This bias is perfectly corrected when the visibilities are calibrated, i.e. when the raw visibility of the science object is divided by the calibrator visibility.

A binning of 6 pixels in the dispersion direction was used to increase the signal, providing a spectral resolution of about 15. The visibilities were calibrated and multiplied by the flux calibrated spectra shown in Fig. 11. The aperture 5 is placed at the maximum of correlated flux which coincides with the peak of the deconvolved $8.7 \mu \mathrm{m}$ image.

\section{Description of the images}

Our NACO observations cover at unprecedented spatial resolution the spectral region of transition between the optical and near-IR, where the image is dominated by scattering processes. The MIDI observations cover the mid-IR thermal emission from the dust regions. The general appearance of the $J, H$, $K$ s images is indeed very different from that of the $L^{\prime}$ and the $8.7 \mu \mathrm{m}$ images.

In the near-infrared, the central $11^{\prime \prime} 5$ of the nebula are dominated by a point source. A complex "butterfly" morphology in the immediate vicinity emerges clearly only at around $2 \mu \mathrm{m}$, though some features can already be traced at shorter wavelengths (Fig. 1).

In Fig. 7, we have labelled the structures seen in our deconvolved NACO $3.74 \mu \mathrm{m}$ image in order to guide the discussion on the geometry of the dusty nebula.

\subsection{Weigelt blobs and other dusty clumps}

The core (central $1^{\prime \prime} \times 1^{\prime \prime}$ aperture) is dominated in the northwest by a region of large dust content which was not resolved by Smith et al. (2003a) but is clearly visible in our NACO data. We call this region the "Weigelt" complex (Weigelt \& Ebersberger 1986; Hofmann \& Weigelt 1988). The Weigelt complex dominates the stellar flux in the mid-infrared, shifting the core photocenter from the star to about $0 . ' 3$ north, as seen by the difference between the raw and deconvolved MIDI images at $8.7 \mu \mathrm{m}$ (Figs. 10 and 9). The Weigelt blobs are small, dense knots of dust and gas northwest of the star within about $1000 \mathrm{AU},(0$. 3).

The changes in position and hence the velocities of the Weigelt blobs were studied by further speckle observations about 10 years after the initial ones (Weigelt et al. 1995, 1996) and also by HST imaging and spectroscopy (Davidson et al. 1997; Dorland et al. 2004; Smith et al. 2004a). These observations demonstrated that the ejecta are moving slowly (less than $50 \mathrm{~km} \mathrm{~s}^{-1}$ from the star) on the equatorial plane. This means that our image of the Weigelt complex seen at $4 \mu \mathrm{m}$ are probably comparable to the optical ones detected some years ago. An attempt of this kind is shown in Fig. 8 by using the images published in Morse et al. (1998).

The position of the largest structures (clumps) can be measured accurately from our images. The brighter clump can be related to the Weigelt clump $\mathrm{C}$, but the clump B is clearly absent. The location of clump D is close but not coincident to a bright clump in the hook-shaped region directly north of the star. The hook is also very close to a structure called the UV knot in the image published by Morse et al. (1998, Fig. 8). It must be stressed out that this comparison is based on images separated in time. However Dorland et al. (2004) have confirmed that the proper motion of these structures is at most $\simeq 5$ mas per year. In a span of 5 years the clumps have moved by at most 25 mas, i.e. less than one pixel on the NACO detector. We are convinced that the structures seen in the NIR are correlated to the ones seen in the visible or UV. The visible structures, dominated by scattering, trace the walls of the dense clumps of dust. We propose to call the two brightest NIR clumps to the north-east of the star clumps $\mathrm{C}^{\prime}$ and $\mathrm{D}^{\prime}$ since they do not coincide with the visible ones but are probably related to them.

Astrometric measurements of the Weigelt blobs C and D have been recently reported by Dorland et al. (2004) and by Smith et al. (2004a). Both used HST data to measure the relative proper motions of blob $\mathrm{C}$ and blob $\mathrm{D}$ with respect to the star. The position angles are consistent with linear, radial, ballistic motions and no evidence of azimuthal motion was detected. The weighted position angle measurements from Dorland et al. for blobs $\mathrm{C}$ and $\mathrm{D}$ are $\mathrm{PA}_{\mathrm{C}}=300.6 \pm 0.6$ and $\mathrm{PA}_{\mathrm{D}}=336.8 \pm 0.4$ degrees. Dorland et al. used a least-squares three-parameter fit of a two-dimensional Gaussian with fixed width and removed the strong diffusive background by using a median filter method.

We applied a similar method on our NACO images, i.e. on the two deconvolved images of the narrow band filters at $3.74 \mu \mathrm{m}$ and $4.05 \mu \mathrm{m}$ and on the $K \mathrm{~s}$ image (without median filtering). This method was not possible for the saturated $L^{\prime}$ filter. We also used a two-dimensional Gaussian for the fits with the $F W H M$ taken as a free parameter. These measurements are a starting point to a further monitoring of these structures by NACO. We decided to concentrate on the two brightest blobs $\mathrm{D}^{\prime}$ and $\mathrm{C}^{\prime}$ and on the bright southern clump that we call SE (Fig. 7). The results are presented in Table 3.

While Dorland et al. and Smith et al. propose different ejection dates for the clumps of 1941 and 1890 , respectively, their measured values of $1934 \pm 20$ and $1907 \pm 12$ agree within statistical errors. In visible and UV spectral regions, the blob emission is dominated by the ablated halo while the dusty clumps are traced by NACO. The consequence is a large variability in shapes and hence centroid positions of the blobs as seen with different filters. NACO has the great advantage of providing the location of the dust clumps in the NIR with a 
spatial resolution comparable to that of the HST. The contribution of the scattered light in $L^{\prime}$ is drastically reduced relative to shorter wavelengths. NACO measurements are not yet able to provide further constraints of the outburst but soon will be. More effort should be put in to decreasing the error bars of single position measurements by using different methods of position determination, as shown by Smith et al. We thus advocate a monitoring of the Weigelt complex by NACO at least during a span of 6 years which would correspond also to the course of the 5.52-year motion of the binary.

\subsection{Arcs and filaments}

The geometrical aspect of the dusty nebula is impressive. It is characterized in both the NACO and MIDI images by two particularly dark regions in the east and south-west, and a third one in the north-east where a faint nebulosity is visible, suggesting that these regions are also relatively devoid of dust.

The resemblance between the MIDI deconvolved image (Fig. 9) and the NACO one is striking. A large part of the regions denoted in Fig. 7 can be recognized (some of them interrupted by the limits of the MIDI FOV): the Weigelt complex, the NE and SW regions, the Northern and Western arcs, the S clump.

It must be pointed out that the brighter clumps discussed in the previous section are just the emerged part of a fainter nebulosity contained within well-defined borders of about $0.5 \times 0.5$ shown in Fig. 7. This triangle-like nebulosity seems to be connected to the south with a fainter structure, the "SE filament" apparently aligned along the same axis as the Weigelt blobs complex $\left(\mathrm{PA} \sim 300-330^{\circ}\right)$. There is no clear separation between the bright northern Weigelt blob complex and this SE filament. Moreover, the Weigelt complex clearly embeds the star itself and the most probable explanation of the faintness of the SE filament is the small amount of material involved in this structure. The Weigelt complex appears interrupted just to the north-east of the star, giving birth to a "hook" region directly to the North, reminiscent of the one detected in UV by Morse et al. (1998, see also Fig. 7).

Of particular interest is the bright spot at about $0.5-0 .{ }^{\prime} 8$ southeast of the star, seen particularly well in our $8.7 \mu \mathrm{m}$ image (also see Smith et al. 2003a) and in the $L^{\prime}$, Pf $\gamma$ and Br $\alpha$ images. This blob connects two well-defined arcs: the Southern arc and the SE arc. The SE arc, brighter in the NIR, has already been denoted as "jet" from images at lower resolution (see Rigault \& Gejring 1995; or Fig. 1 in Smith \& Gehrz 2000, for instance).

The Southern and SE arcs seem to partly hide the SE filament and seem to be in front of it. Moreover they are connected in a complex but traceable way to the northern arcs. These arcs are apparently hidden (or embedded) in the north by the Weigelt complex. This is particularly visible in the $3.74 \mu \mathrm{m}$ and $4.05 \mu \mathrm{m}$ images (Fig. 5), but some hints can also be extracted from the MIDI image.

\subsection{Dust sublimation radius}

Our NACO Pf $\gamma$ and $\operatorname{Br} \alpha$ deconvolved images show no evidence for significant emission in the inner regions (see Fig. 6). The radius where the flux inflexion point is located is about
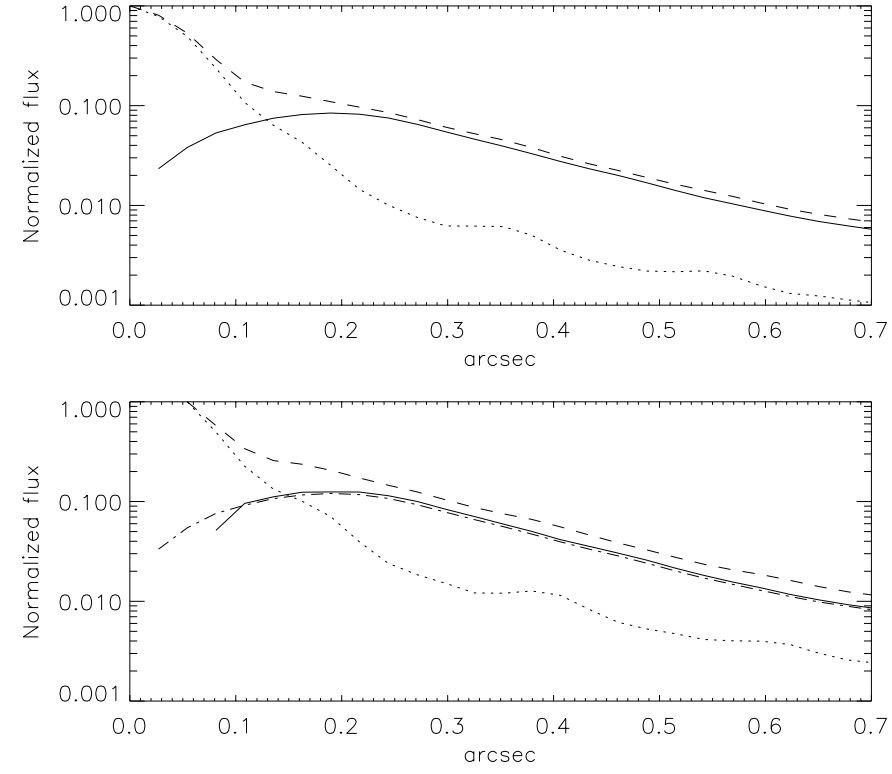

Fig. 6. On the top, radial flux normalized to the peak with NB_3.74 filter for Eta Car (dashed line), and the PSF HD 101104 (dotted line) and their subtraction (solid line). Close to the source, a strong decrease of the flux is clearly seen that we attribute to the dust sublimation region at about 0.1 (230 AU). On the bottom panel the same treatment is applied to the NB_4.05 images. However, the flux reference is chosen to be at $\sim 0$.'05 ( 2 pixels) from the peak in order to account for the non-linearity of the detector at maximum. The dashed-dotted curve is the residual of the NB_3.74 filter subtraction scaled for comparison. The two curves agree fairly well and the contribution of the $\operatorname{Br} \alpha$ line is hardly visible.

130-170 mas in both deconvolved images. The empty regions are not symmetric around the source; they are more extended to the north than to the south. It could be argued that this gap is an artefact of the deconvolution process but a decrease of emissivity is already visible in the radial profiles shown in Fig. 6, extracted from the raw images. We have carried out further tests to verify the reality of the feature. While increasing the number of iterations by steps, we checked that at each time the gap remained stable in size and shape. Until the appearance of strong artifacts affecting the whole image, this feature behaves like any other: its shape is slowly distorted, but its position remains essentially unaffected. The same behavior is seen in the deconvolution of both the Pf $\gamma$ and the $\operatorname{Br} \alpha$ images, but the artifacts appear earlier for the slightly overexposed $\operatorname{Br} \alpha$ image.

Indeed, the presence of a gap of this size does not contradict our other knowledge of Eta Car. The deconvolved Pf $\gamma$ and $\operatorname{Br} \alpha$ images reach a spatial resolution of about 60 mas. This scale is particularly interesting since it is close to the expected radius where dust sublimates.

From the approximation of a black-body equilibrium temperature, Smith et al. (2003a; Eq. (3)) use the following formula for the disk temperature:

$T_{\text {dust }} \simeq 13100 \times R_{\mathrm{AU}}^{-1 / 2} \mathrm{~K}$.

We see that a dust temperature of about $400 \mathrm{~K}$ is expected at $900 \mathrm{AU}$ or 0.'4 (if shielding from closer material is not taken into account). Taking a typical sublimation temperature 


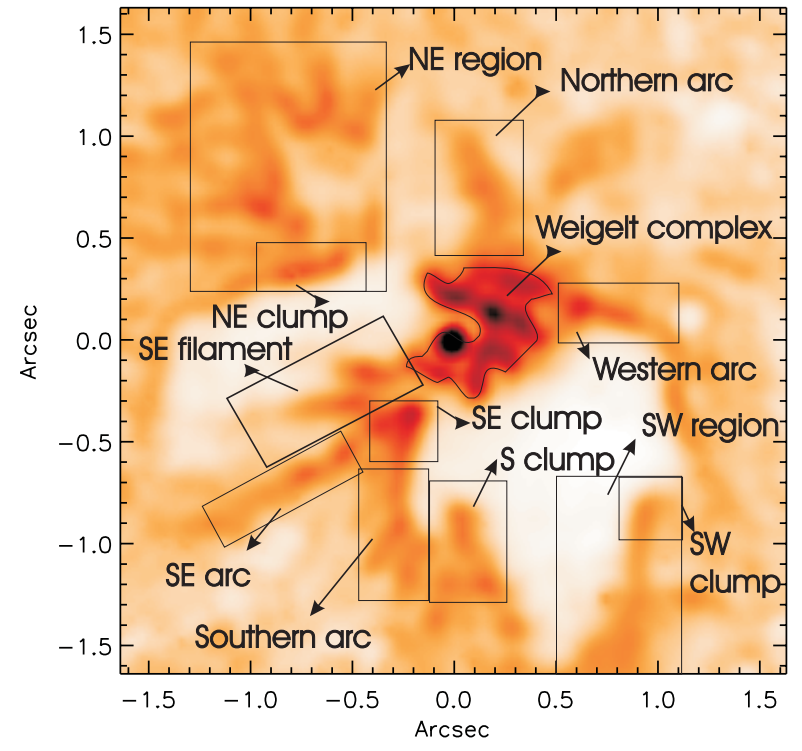

Fig. 7. Location of interesting regions in the "butterfly" dusty nebula close to Eta Car. The naming convention is partly based on Fig. 4 of Smith et al. (2003a).

of about $1000 \mathrm{~K}$, we find that dust is not expected within a radius of about $150 \mathrm{AU}$, i.e. 70 mas from the central star. Of course, the dust sublimation radius is only an indicative distance; the amount of dust will steadily decrease as the temperature increases beyond the sublimation point of each dust species. In the Sect. 4, we provide some indications on the dust composition in the nebula.

This gap between the central source and the Weigelt complex may have another explanation. Dorland et al. (2004) and Smith et al. (2004a) have presented evidence that the Weigelt blobs C and D were created in an outburst, either in 1941, or in 1890. If no dust has formed in the equatorial plane since then, then the gap is a natural consequence of the proper motion of the Weigelt blobs and not related to the temperature near the central star.

The question of the vertical extent of the Weigelt complex is also a difficult one. Hillier \& Allen (1992) argued that the central source is extinguished by dust, while the Weigelt blobs suffer much less circumstellar extinction. The location of this obscuring material is somewhat uncertain. Why should the star be occulted, but not the Weigelt blobs? Moreover the central source has brightened appreciably over the last decade. At V it is now a factor of 3 brighter (see Davidson et al. 1999, and recently Martin et al. 2004). The simplest interpretation is that the extinction is decreased, and hence dust is evaporating, leading to a larger void region around the star. Interestingly, van Genderen \& Sterken have shown that this brightening occurred in a relatively short time after the 1998.0 spectroscopic event attributed to the periastron passage a hot companion (see also Sect. 6.3).

In the deconvolved images (see Fig. 5) a large part of the nebulosity has disappeared in the treatment, but in Fig. 2 we can see that the star is somewhat embedded. In $L^{\prime}$ the dust becomes more and more optically thin and the regions towards the line of sight are difficult to detect. This can be done only by a careful study involving several filters, to map the extinction and evaluate the amount of scattering. This study could be performed with carefully calibrated NACO images but this implies dedicated observations which will be postponed to a future study.

\section{Dust composition and temperature}

In this section we will concentrate on the interpretation of the 9 single-dish $N$ band MIDI spectra. The $N$ band spectra of Eta Car are characterized by a strong, smooth feature around $10.5 \mu \mathrm{m}$. The feature has an unusually broad wing at the long wavelength side. The 9 MIDI spectra, shown in Fig. 10, display a change in the emission feature as a function of position in the nebulae. From the north to the south the peak position is shifted from 10.5 to $11.5 \mu \mathrm{m}$.

In order to study the mineralogy of the dust we made an attempt to fit the $N$-band spectra. The spectrum in the $10 \mu \mathrm{m}$ region is dominated by thermal emission from warm $(T>$ $250 \mathrm{~K})$, small ( $a<2 \mu \mathrm{m})$ dust grains. Colder grains will emit most radiation at longer wavelengths while big dust grains will contribute mainly to the continuum which makes the determination of their mineralogy difficult. We use here a very simple model consisting of a single blackbody source function with two different dust species, amorphous olivine $\left(\mathrm{MgFeSiO}_{4}\right)$ and corundum $\left(\mathrm{Al}_{2} \mathrm{O}_{3}\right)$. We also add continuum emission with the same temperature. The choice of the dust components will be discussed below. We take a single grain size of $0.1 \mu \mathrm{m}$. Using a more complicated source function involving a distribution of temperatures or by including more dust species or grain sizes did not improve the fit significantly. By including more dust species we find that some trace of crystalline olivine might be present but with an abundance less than $5 \%$. In order to calculate the emission efficiencies of the dust grains, we have to assume a shape of the dust grains. The choice of the particle shape model can be crucial in obtaining reliable results. However, since both dust components used here have a rather smooth behavior we restrict ourselves to simple, frequently used methods to calculate the emissivities. The best fitting results were obtained if we take the amorphous olivine grains to be homogeneous and spherical. For the corundum grains we had to use a so-called continuous distribution of ellipsoids (CDE) (Bohren $\&$ Huffman 1983) to reproduce the observations. The abundances are obtained by using a standard linear least square fitting procedure. This simple model gives us an indication of the composition of the small, warm dust component, and, using the observed MIDI spectra, provides a quantitative way to study the spatial variation in the dust composition.

We include amorphous olivine in our fitting procedure because it is one of the most abundant dust species in circumstellar, cometary and interstellar dust. Corundum is expected to be the first species to condense at very high temperatures $(1700 \mathrm{~K})$ and preferably at high densities (corresponding to a pressure of $10^{-3}$ atm, see e.g. Tielens 1990). Mitchell \& Robinson (1978) showed that a substantial amount of corundum is needed in order to fit the large aperture $10 \mu \mathrm{m}$ spectrum of Eta Car. The spectrum of Eta Car obtained by ISO, presented by Morris et al. (1999), provides additional evidence for the presence 

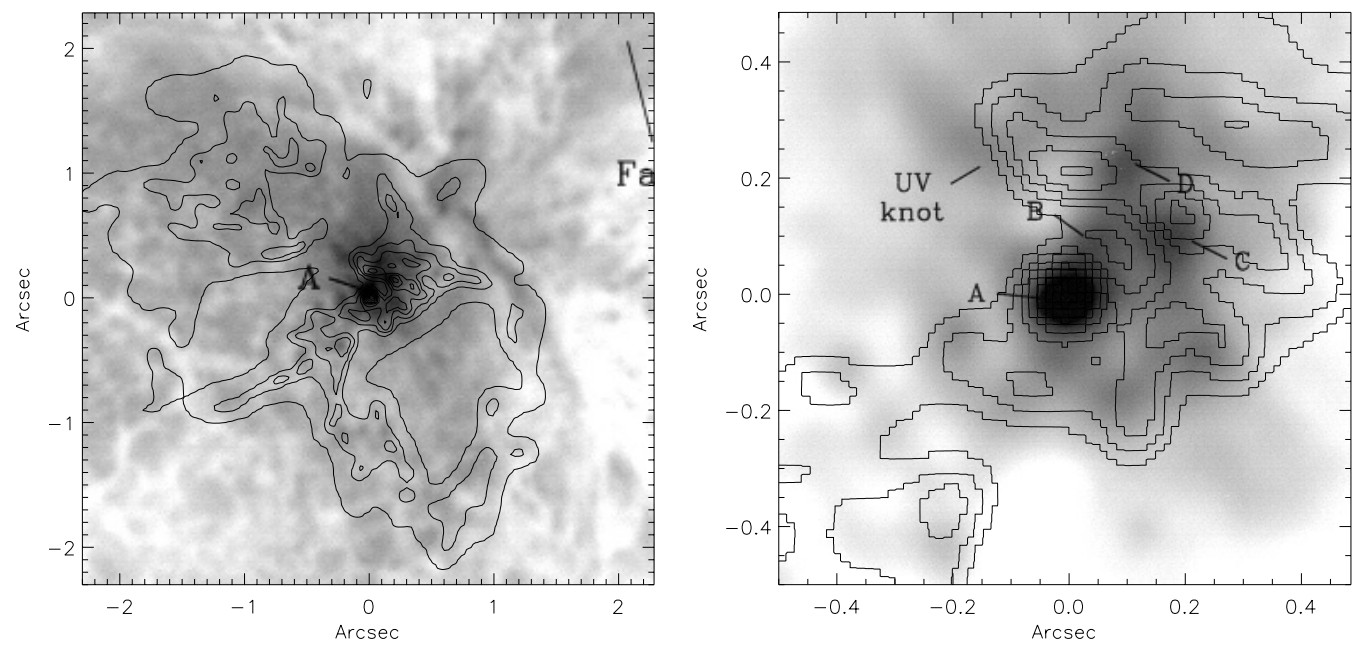

Fig. 8. Comparison of the $3.74 \mu \mathrm{m}$ deconvolved NACO image (in contours) with the highest resolution HST images (background, from Morse et al. 1999) at large (left) and small (right) scales. The location of the dusty clumps does not coincide with that of the Weigelt blobs, but their structure is somehow complementary to the image. The optical/UV blobs are probably hot regions less shielded from the central star's UV flux which could coincide with the dust clump surfaces facing the star. This is particularly true for Weigelt blobs C and D.

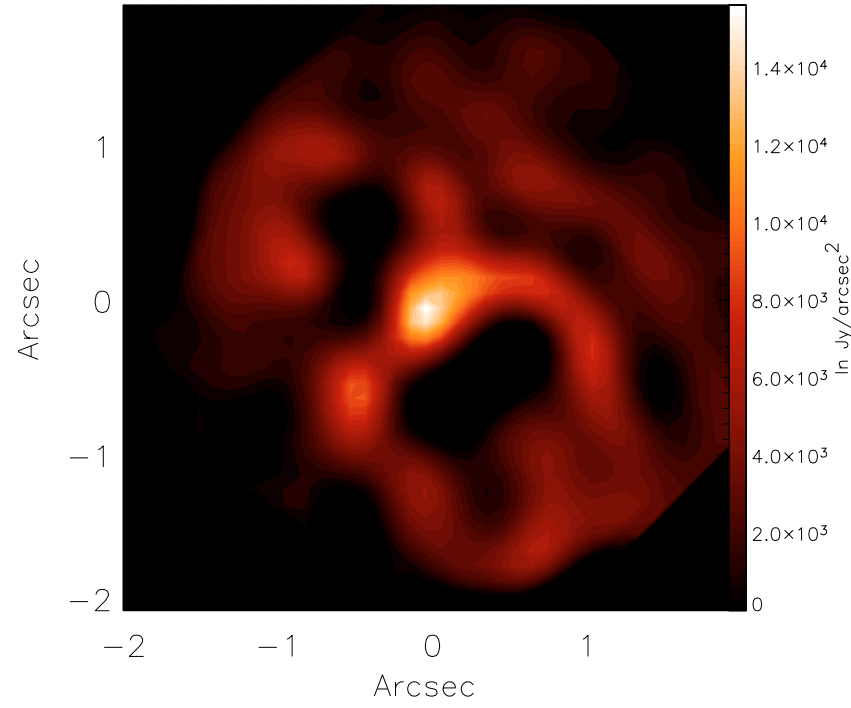

Fig. 9. Deconvolved acquisition image of Eta Car with the $8.7 \mu \mathrm{m}$ filter (UT3). In order to enhance the contrast, the image $\mathrm{I}^{1 / 4}$ is shown but the color scale is expressed in Jy/ $\operatorname{arcsec}^{2}$. The bright spot which emerges in this image is in the position of the central star and at the location where a strong correlated flux has been detected by MIDI (see Fig. 12). The image has been de-rotated with respect to Fig. 10 so that the north is up and east is left.

of non-silicate dust. The reason is that if all of the emission around $10 \mu \mathrm{m}$ were caused by silicates, they would generate an appreciable $18 \mu \mathrm{m}$ emission feature, which is not observed. Moreover, the broad red wing of the $10 \mu \mathrm{m}$ feature extends to $15.5 \mu \mathrm{m}$, which is not characteristic of silicate emission. Another argument that the $10 \mu \mathrm{m}$ emission should contain a significant component of non-silicate dust is the lack of a clear detection of crystalline silicates as seen in some other LBVs (Waters et al. 1997). As it is hard to explain that in Eta Car only amorphous silicates would form, the lack of observable silicate crystals suggests that the dust material is not completely dominated by silicates. Finally, we note that corundum is also

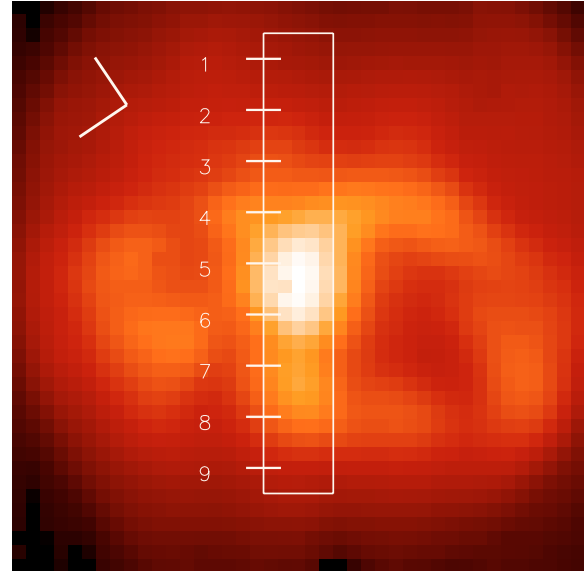

Fig. 10. MIDI $8.7 \mu \mathrm{m}$ acquisition image. The north points towards the upper-left side and east the lower-left and the position of the slit is indicated. The slit is $0{ }^{\prime \prime} 6$ wide and $3^{\prime \prime}$ long. The numbers indicate the central positions of the apertures described in Table 4.

expected to be present in the ambient environments of these types of stars. In recent studies of AGB stars for instance, abundances of corundum of about $10-30 \%$ are reported (see the extensive discussion in Maldoni et al. 2004). Moreover, the appearance and disappearance of the corundum signature in the variable spectra of pulsating $\mathrm{OH} / \mathrm{IR}$ stars is interpreted as evidence for dust formation (Maldoni et al. 2004). The above arguments strongly favor a grain component in addition to silicates. As corundum can naturally explain the red-wing of the $10 \mu \mathrm{m}$ feature, we adopt this species as the extra component.

It is worth discussing that Mitchell \& Robinson (1986) discarded the possibility of corundum as a dust component. They did so because i) corundum is not expected to survive as a separate grain component as it would act as nucleation centers for the later precipitation of silicates; and ii) the required amount of aluminum suggested an unrealistic overabundance compared to the solar value. Mitchell \& Robinson fitted the broad long-wavelength shoulder of the $10 \mu \mathrm{m}$ feature (which 

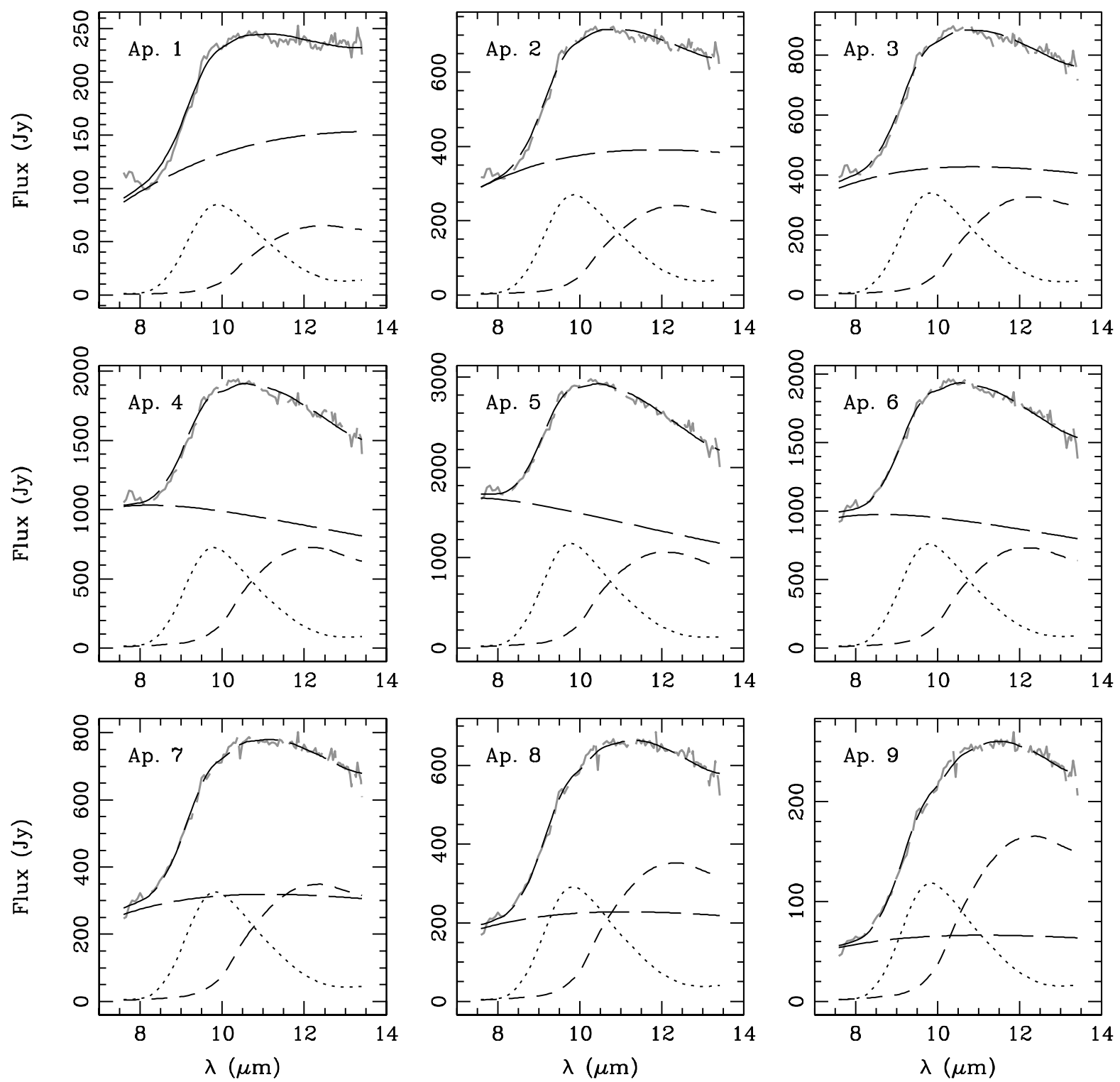

Fig. 11. Spatially resolved MIDI spectra expressed in Jansky (grey lines) together with the spectra of the best fit models (solid line). The dotted line shows the olivine contribution, the dashed line the corundum contribution and the long-dashed shows the continuum emission. The spectra are extracted from the north-west (upper left panel) to the south-east (lower right panel). The slit is aligned to the nebula. The 9 spectra are spaced by 0.4 , the maximum flux in the MIR is in aperture 5 while the star is located in aperture 6 . The SE clump and the southern arcs spectra are in apertures 7,8 and 9.

we attribute to corundum, see below) by emission from large $(2 \mu \mathrm{m})$ amorphous silicate grains that display a very broad $10 \mu \mathrm{m}$ feature. For their calculations they use the refractive indices of "astronomical silicate" as derived by Draine \& Lee (1984). However, calculations for large amorphous olivine grains using laboratory measurements of the refractive indices, (e.g. Dorschner et al. 1995), show a feature that is less broadened and is incompatible with the observed red wing of the $10 \mu \mathrm{m}$ MIDI spectra. Concerning their first argument, one should keep in mind that the Eta Car nebula is expected to be a CNO processed medium (Davidson et al. 1986; Waters et al. 1997; Smith \& Morse 2004). This could lead to a condensation sequence that is different from that which occurs in other stars favoring the creation of corundum. In Eta Car, the amount of oxygen that remains after the $\mathrm{CO}$ molecule formation could be so modest relative to that of the metals that part of the material is only able to form simple oxides, such as $\mathrm{Al}_{2} \mathrm{O}_{3}$. (Also, one may expect a chemistry driven by remaining metals, notably sulphur, forming species such as MgS.) An alternative explanation for the presence of corundum may be that the gas density at the location of dust formation is so low that it is not possible to complete all of the condensation sequence leading to silicate dust, i.e. the condensation reactions freeze out. Concerning the second argument of Mitchell \& Robinson, note that both possibilities discussed above may, at least in principle, explain the apparent over abundance of solid state aluminum relative to silicon.

The results of the analysis as described above are summarized in Table 4, the 9 spectra are shown in Fig. 10 together with the best fit model. Also shown are the contributions from 

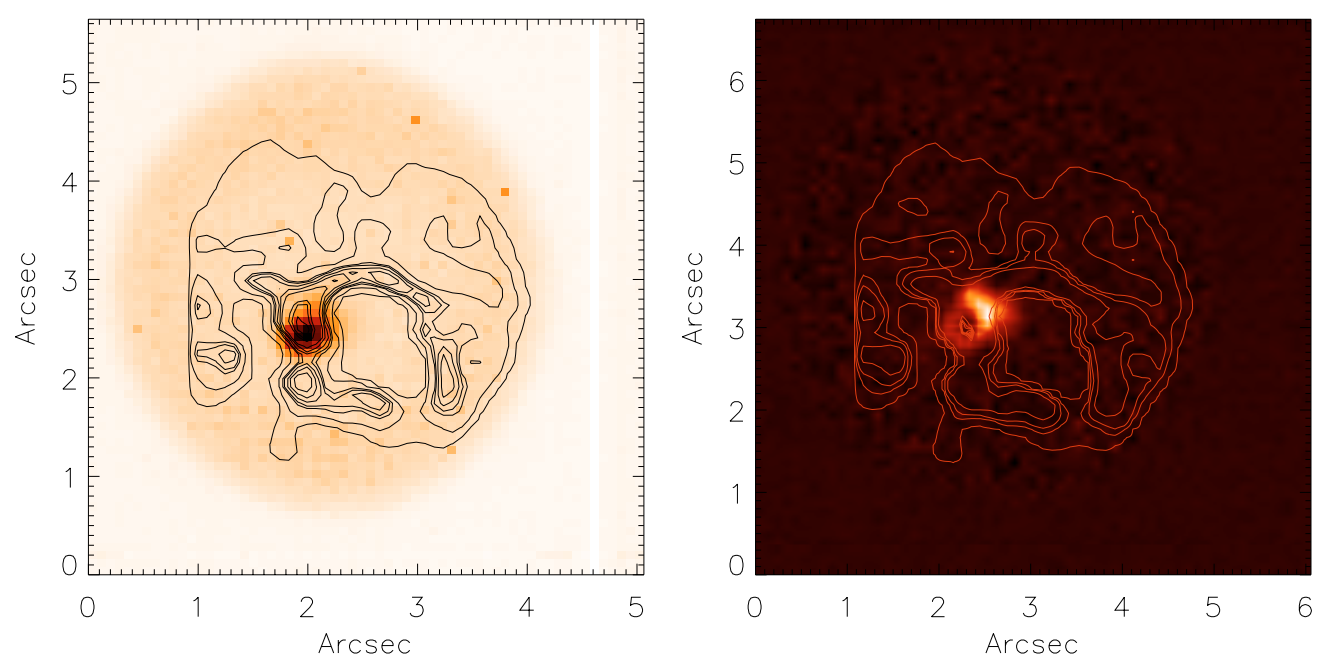

Fig. 12. Left, the figure shows the rms of the fluctuations within the MIDI FOV. The external regions are dominated by the detector noise and the internal regions by the tunnel and sky background fluctuations. The signal from the fringes is strong and centered on the position of the star as seen in the deconvolved acquisition image at $8.7 \mu \mathrm{m}$. The contour plot represents the contours of the deconvolved MIDI $8.7 \mu \mathrm{m}$ acquisition image. Right, the noise pattern and the fringe pattern from a calibrator have been subtracted from the previous figure in order to show the extended fringe signal. The orientation is the same as in Fig. 10.

Table 3. Separation and position angle measurements with respect to the star of several blobs seen in our images.

\begin{tabular}{|c|c|c|c|c|c|c|}
\hline \multirow[b]{2}{*}{ Image } & \multicolumn{2}{|c|}{ Blob C'/C } & \multicolumn{2}{|c|}{ Blob D'/D } & \multicolumn{2}{|c|}{ Blob SE } \\
\hline & $\begin{array}{c}\text { Separation } \\
\text { (mas) }\end{array}$ & $\begin{array}{c}\text { PA } \\
(\mathrm{deg})\end{array}$ & $\begin{array}{c}\text { Separation } \\
\text { (mas) }\end{array}$ & $\begin{array}{c}\text { PA } \\
(\mathrm{deg})\end{array}$ & $\begin{array}{l}\text { Separation } \\
\text { (mas) }\end{array}$ & $\begin{array}{c}\text { PA } \\
(\mathrm{deg})\end{array}$ \\
\hline$K \mathrm{~s}$ & - & - & - & - & $379 \pm 4$ & $120.0 \pm 2$ \\
\hline NB_374 & $233 \pm 13$ & $300 \pm 15$ & $218 \pm 14$ & $352 \pm 17$ & $418 \pm 13$ & $121.9 \pm 3$ \\
\hline NB_405 & $237 \pm 9$ & $298 \pm 11$ & $216 \pm 9$ & $359 \pm 14$ & $401 \pm 21$ & $123.4 \pm 3$ \\
\hline $8.7 \mu \mathrm{m}$ & - & - & - & - & $603 \pm 38$ & $136 \pm 12$ \\
\hline$F 550 M^{1}$ & $224 \pm 3$ & $299 \pm 3$ & $259 \pm 3$ & $335 \pm 3$ & - & - \\
\hline
\end{tabular}

the various components. We see that when we go down from north to south the abundance of corundum is increased. This is consistent with the observed shift of the feature towards longer wavelengths when going from north to south.

It should be noted that the derived abundances are subject to a correct estimate of the continuum contribution. In our simple model the contribution from cold dust grains to the continuum emission is not taken into account. Including this in a more complicated model might cause changes in the derived dust composition. To test the effect of grain size on the derived abundances we have performed calculations in which large amorphous olivine grains were added. This reduced the derived abundance of corundum in all fits by $\sim 5 \%$, i.e. the trend in the compositional gradient is not significantly effected.

The evolution seen in the spectra is indirect evidence that dust is continuously created in the butterfly nebula or at least that the geometry of the butterfly nebula strongly influences the chemical composition of its dust content. The aperture $1 \mathrm{spec}-$ trum is dominated by emission from olivine grains. This aperture is pointed towards the region where the Weigelt complex ends and probably encounters, in the equatorial plane, the walls of the polar lobes. The dust in this region is efficiently shielded from the light of the central object. The aperture 7-9 spectra are dominated by the emission from aluminum oxide grains. A possible explanation for this might be that the condensation reactions freeze out. In this scenario the difference in dust composition between the Weigelt complex and the SE clump reflect a different formation process; the equatorial dust being formed preferably during outbursts which provide dense enough regions to complete the condensation process whereas the dust formed in the rims of the butterfly nebula is continuously processed but the reactions quickly freeze out. An argument against this scenario, however is that the impact of the wind on the rims should provide a density discontinuity large enough to provide the conditions for dust silicate formation. Spectra taken beyond the rims of the butterfly nebula are needed to constrain the condensation sequence and begin a study of the chemical map of the dust within the full nebula.

\section{Correlated flux}

\subsection{Location of the fringes}

The spatial distribution of the fringes detected by MIDI with the $8.7 \mu \mathrm{m}$ filter is shown in Fig. 12. We have checked that 
Table 4. Positions and beam size of the apertures used to extract the spatially resolved spectra along the main axis of the nebula, increasing numbers from north to south. The three last columns report the result of the best fit to the spectra by using thermal emission with temperature $T_{b b}$ and opacities computed for various dust species.

\begin{tabular}{ccc|ccc}
\hline \hline Ap. & $\begin{array}{c}\text { Shift } \\
(\mathrm{mas})\end{array}$ & $\begin{array}{c}F W H M \\
(\mathrm{mas})\end{array}$ & $\begin{array}{c}T_{b b} \\
(\mathrm{~K})\end{array}$ & $\begin{array}{c}\text { Silic. } \\
(\%)\end{array}$ & $\begin{array}{c}\mathrm{Al}_{2} \mathrm{O}_{3} \\
(\%)\end{array}$ \\
\hline 1 & -1568 & 350 & $310 \pm 50$ & $75 \pm 15$ & $25 \pm 15$ \\
2 & -1176 & 330 & $390 \pm 50$ & $65 \pm 10$ & $35 \pm 10$ \\
3 & -784 & 200 & $460 \pm 50$ & $60 \pm 10$ & $40 \pm 10$ \\
4 & -392 & 200 & $600 \pm 70$ & $55 \pm 5$ & $45 \pm 5$ \\
5 & 0 & 200 & $720 \pm 80$ & $55 \pm 5$ & $45 \pm 5$ \\
6 & 392 & 200 & $570 \pm 5$ & $55 \pm 5$ & $45 \pm 5$ \\
7 & 784 & 200 & $440 \pm 50$ & $55 \pm 5$ & $45 \pm 5$ \\
8 & 1176 & 330 & $440 \pm 50$ & $50 \pm 5$ & $50 \pm 5$ \\
9 & 1568 & 350 & $480 \pm 60$ & $45 \pm 5$ & $55 \pm 5$ \\
\hline
\end{tabular}

the peak of the fringes is localized at the position of the star itself ${ }^{1}$ but an extended halo is also visible in the Weigelt complex about $0 .{ }^{\prime} 4-0{ }^{\prime} 6$ northwest from the star. This is the confirmation that highly compressed material emitting strongly at $8.7 \mu \mathrm{m}$ exists in this region.

The fluctuations from the fringes at the location of the Weigelt blobs are definitely more extended than a single PSF $F W H M$ at $8.7 \mu \mathrm{m}$ (220 mas). They coincide roughly with the location of the blob $\mathrm{C}^{\prime}$ observed by NACO but are more extended owing to the larger PSF of the $8 \mathrm{~m}$ telescope at this wavelength. This implies that in the equatorial Weigelt region a fraction of the dust is embedded in clumps with a typical size smaller than 10-20 mas (25-50 AU) within a total extent of about $1000 \mathrm{AU}$. Nevertheless, this correlated flux represents only a few percent of the total flux at these locations. It must be pointed out that only a few scans with fringes have been recorded during this commissioning measurement and the lowest detectable fringe signal visible in Fig. 12 is about $20 \mathrm{Jy}$. In June, the measurements performed in dispersed mode (following section) represents more than 200 scans. MIDI has been able to record fringes further out from the central source with a sensitivity reaching about $5 \mathrm{Jy}$. From this result we are confident that the spatial distribution of the correlated flux can be studied in the future at distances larger than 0.5 from the central object.

\subsection{Correlated spectra}

In Fig. 13, we show the correlated flux of three central masks around the star (apertures 5 to 7). For comparison, the photometric flux of aperture 6 is shown (this aperture contains the star, the maximum flux being in aperture 5). The correlated fluxes measured by MIDI are $98 \pm 47 \mathrm{Jy}, 87 \pm 33 \mathrm{Jy}$ and $82 \pm 41 \mathrm{Jy}$, respectively. The errors have been estimated from

\footnotetext{
${ }^{1}$ By comparing the position of the fringes with the position of the emerging peak in the deconvolution image (Fig. 9).
}

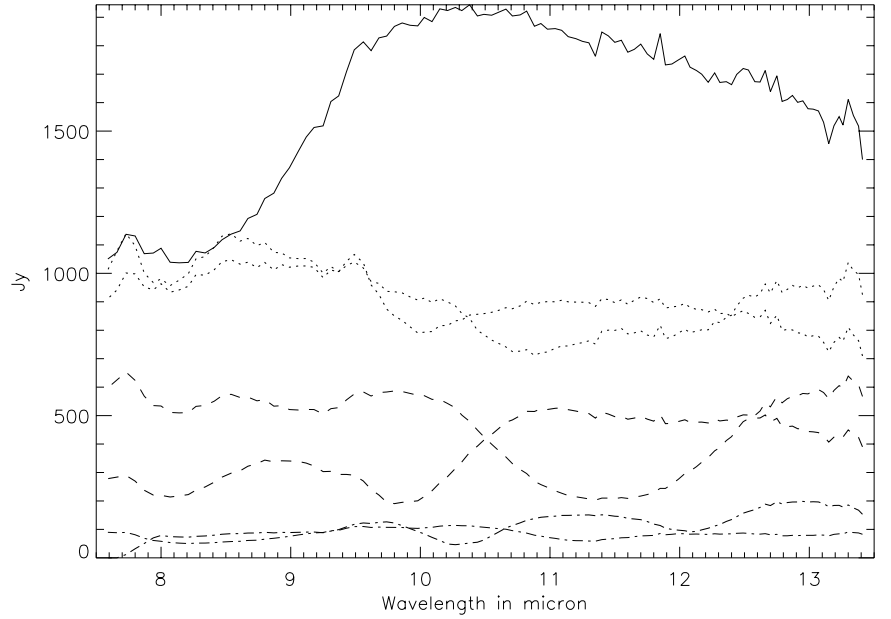

Fig. 13. MIDI correlated flux measured with a $74 \mathrm{~m}\left(\mathrm{PA}=62^{\circ}\right)$ and a $78 \mathrm{~m}\left(\mathrm{PA}=56^{\circ}\right)$ projected baseline. The solid line denotes the photometric flux as extracted using the mask centered on the star (aperture 6) which can be seen in Fig. 11. The dotted lines represent the correlated flux measured with the aperture 6 . scale has been multiplied by 10. The dashed and dashed-dotted lines represent the correlated flux extracted with aperture 5 (dashed) and 7 (dashed-dotted).

the variance of the measurements using several calibrators and by varying slightly the parameters of the apertures. The fringes have been recorded using the same slit as used for the photometry. Unfortunately, this slit is about two PSFs wide and the signal from the star has been mixed up with the signal from the dust situated perpendicular to the axis, i.e. at $\mathrm{PA} \simeq 60^{\circ}$.

The baseline is roughly perpendicular to the main axis of the nebula and van Boekel et al. (2001) have reported that the star is prolate. This means that the baselines were oriented perpendicular to the main stellar axis, where the star is smaller, corresponding to a maximum correlated flux. Hence, our measurement can be considered as an upper limit of the correlated flux observable from the star.

We compared these measurements with the model presented in Hillier et al. (2001). For that purpose, we used three flux distributions from the model at 8,10 and $13 \mu \mathrm{m}$, of respectively 332, 287 and $241 \mathrm{Jy}$ which can be approximated by a 2D Gaussian with a $F W H M$ equal to 6.4, 6.8 and 8.2 mas. With the UT1-UT3 projected baseline of $78 \mathrm{~m}$, we can compute the expected correlated flux by performing the Fourier transform of the flux distribution from the (spherical) models. The visibility for the theoretical star at 8,10 and $13 \mu \mathrm{m}$ is $0.54,0.59$ and 0.65 respectively. This corresponds to correlated fluxes of 180, 169 and $156 \mathrm{Jy}$. These fluxes are larger than those observed by a factor of 2 . Moreover, the correlated flux measured at the location of the central star includes also a non-negligible contribution from the dust.

The correlated flux from the Weigelt region is dominated by the brightest clumps. Due to the complexity of their spatial distribution, the curves of correlated flux present an oscillating behavior which is very dependent on the projected baseline length and direction. This is particularly visible in the correlated flux spectra extracted from aperture 5 (dashed line in Fig. 13). In particular, the frequency and the stability of this oscillation over 
the $N$ band suggest that a few clumps separated by $0 . ' 05-0{ }^{\prime} 1$ dominate the correlated flux of apertures 5 and 7 . The correlated flux extracted from aperture (which contains the star) is larger and the oscillation much lower suggesting that the dust contribution is relatively low, about 10-20 Jy, compared to the stellar flux. We are left with a stellar flux of about 70-90 Jy at $8 \mu \mathrm{m}$ and about 50-70 Jy between 10 and $13 \mu \mathrm{m}$.

The correlated fluxes represent about $50 \mathrm{Jy}$ in the location of the Weigelt complex and only 5-10 Jy in the south. If we compare these correlated fluxes with the total measured fluxes, the visibility and hence the clumping factor are larger at the location of the Weigelt complex (more than $3 \%$ visibility) than at the SE clump (less than 2\%) though this difference is within the MIDI error bars. We are quite confident that even the smallest correlated fluxes reported here are real. No correlated flux can be detected at the northern edge of the slit. At this location, the flux from the nebula is still well above the detection limit of MIDI, which is of the order of one Jansky for faint fluxes. Moreover, MIDI has observed some bright overresolved sources without showing spurious fringe detection. For instance, no fringe signal was detectable for the bright source $\mathrm{OH} 26.5+0.6$, an $\mathrm{OH} / \mathrm{IR}$ star with a $N$ band flux at the time of our observations of $\approx 650 \mathrm{Jy}$ (Chesneau et al. 2004).

\section{Discussion}

\subsection{Inferences for the observations of the central star}

As noted in Sect. 5.2, the IR fluxes deduced from the present observations, and by van Boekel et al. (2003), are a factor of 2 to 3 smaller than those predicted by the model. Here we discuss the possible cause of these discrepancies.

The first cause we examine is the correction for reddening. From the inferred dust temperatures, and from the $J H K L$ variability observations of Whitelock et al. (2004), we can infer that the $K$ band flux of Eta is dominated by the central source, and by scattering. Feast et al. (2001) give an IR magnitude for Eta Car of around 0.4 to 0.5. This, and the stellar $K$ magnitude of 1.2 derived by van Boekel, implies that half the starlight is scattered. Thus there is considerable extinction at $K$, and this extinction could easily explain the difference between the van Boekel $K$ flux and the model $K$ flux. However at $10 \mu \mathrm{m}$, the extinction will be lower, and probably cannot explain the discrepancy. Moreover, a variable free-free emission seems to be also an important flux contribution in $K$ band which is also contaminated by the emission from the $\mathrm{Br} \gamma$ line (Whitelock et al. 2004). The complexity of the $K$ band is such that the constraints provided in $\mathrm{N}$-band should be more reliable. Thus we must look to the modelling for an explanation of the discrepancy.

There are some major difficulties associated with modelling of the optical/UV spectra of Eta Car.

1. We cannot compare the model fluxes with those observed since the reddening and reddening law are uncertain, and have to themselves be derived from the observations. In addition, the amount of circumstellar reddening is probably variable.
2. There is evidence for a possible wind asymmetry. This might explain explain why Hillier et al. severely overpredicted the strength of the P Cygni absorption lines seen in optical spectra. Direct evidence for an asymmetry comes from the variable terminal velocity derived from the scattered $\mathrm{H} \alpha$ profiles (Smith et al. 2003b), and from VLT measurements (van Boekel et al. 2003).

3. The companion star could be a substantial source of ionizing photons which could also affect the symmetry of the wind. The large impact of the orbital cycle on the nearinfrared photometry is a strong argument for it (Whitelock et al. 2004).

4. The spectrum of the primary is intrinsically variable, and part of the variability is probably not attributable to a companion. In particular during 2002, and leading up to the event in 2003, the $\mathrm{H}$ Balmer lines were up to a factor of 2 weaker compared to the previous cycle. The behavior of the radio emission is also different from the last cycle (Duncan \& White 2003).

Given all these difficulties it is not surprising that the agreement of model and observations is not perfect. The match of the model with a large part of the spectrum from the central object is already a success. However it is worth examining in more details possible causes of the discrepancies. We consider two possible causes: variability and wind asymmetry.

Since the IR flux will originate where the wind is at a substantial fraction of the terminal velocity, we can use the mass-loss rate formula of Wright \& Barlow (1975) to estimate the scaling of the IR flux with mass-loss rate. In particular, $\dot{M} \propto S^{0.75}$. Thus a factor of 2(3) reduction in the IR flux corresponds to a change in the mass-loss rate of a factor of 1.7(2.3). Using HST spectra obtained in March 1998, and assuming $^{2} N(\mathrm{He}) / N(\mathrm{H})=0.2$, Hillier et al. (2001) derived a massloss rate of $1 \times 10^{-3} M_{\odot} / \mathrm{yr}$ with a filling factor of 0.1 . The mass loss rate is primarily derived from the equivalent widths of the Balmer lines, while the filling factor is constrained by the strength of the electron scattering wings. The VLT observations of van Boekel et al. (2003) suggest $\dot{M}=1.6 \times 10^{-3} M_{\odot} / \mathrm{yr}$ with $f=0.225$. However, with this value, the electron scattering wings appear to be somewhat too strong. The flux distributions of the two models are very similar. It is worth mentioning that the HST spectrum of March 1998 has been taken at an orbital phase very close to the one of MIDI measurements. In contrast, the VINCI measurements, have been carried out in the first half of 2002, i.e. at a very different part of the cycle. It is possible in this context that the mass-loss rate and geometry were strongly affected (Smith et al. 2003b).

As noted previously the $\mathrm{H} \alpha$ profiles have changed, and their weakening could be interpreted as a reduction in mass-loss rate. Since the $\mathrm{H} \alpha$ and $10 \mu \mathrm{m}$ emission come from a similar volume (the $\mathrm{H} \alpha$ volume is slightly larger) it is not surprising that a reduction in IR flux accompanies the reduction in $\mathrm{H} \alpha$ flux. An alternative scenario for the variability is that the flux that maintains the ionization of the wind has been reduced. The existence of strong FeII emission lines, the radio variability observations

\footnotetext{
2 In the modelling there is a strong coupling between the derived $\mathrm{H} / \mathrm{He}$ abundance ratio and the mass-loss rate.
} 
(e.g., Duncan \& White 2003), and the models show that H recombines in the outer envelope.

A second explanation is a wind asymmetry. A wind asymmetry will certainly bias our derived mass-loss rates. However a wind asymmetry will generally have substantially less influence on the $K-10 \mu \mathrm{m}$ color, simply because the stellar fluxes at both IR wavelengths are produced by free-free processes, and hence are affected in the same way.

Clearly repeated quasi-simultaneous observations, at 2 and $10 \mu \mathrm{m}$, are very important to ascertain the consistency of the model constraints. This will be possible soon with the advent of (quasi)-simultaneous observations of Eta Car with MIDI in the near-IR interferometer AMBER (Petrov et al. 2003)

\subsection{Geometry of the dusty inner nebula}

The Homunculus shape has been discussed by many authors, but it is only recently that NIR spectroscopy has allowed unambiguous tracing of the shape and orientation of the dense neutral gas and dust through the observation of the $\mathrm{H}_{2}$ emission (Smith 2002). In particular, Smith demonstrates that near the equator the walls of the bipolar lobes do not converge towards the central star (see his Fig. 7). A simple extrapolation of the $\mathrm{H}_{2}$ data indicates that the connection of the lobes with the equatorial plane takes place at about 2000-4000 AU, i.e. about 1'. 3 from the central object. This distance is compatible with the projected mean position of the rims of the dusty inner nebula seen in the NACO and MIDI images. This naturally led Smith et al. $(2002,2003 a)$ to suggest that the complex structures seen in their images indeed lie close to the equatorial plane as has been proven for the Weigelt complex. They tentatively explain the complicated shape of this equatorial structure in the frame of a preexisting torus disrupted during the great eruption of 1840 or by the post eruption stellar wind. The Weigelt complex, which also lies on the equatorial plane would have been ejected later on, in the second eruption of 1890 .

It is indeed very difficult from images only, and without any kinematic information from the structures, to get a 3D view of the object. Within this context any model will be highly conjectural, yet we propose in this section some arguments suggesting another point of view. The images show a highly structured butterfly shape which is well delimited by bright rims. In particular, we have shown that the SE clump is the warm head of a protruding region linking the SE and Southern arcs which exhibits a large amount of corundum. The SE clump seems to be closely aligned with the polar axis of the star and the bipolar nebula. This is for us an indication that this structure could directly face the fast and dense wind of Eta Car, and therefore not lie in the equatorial plane. The rims of the dusty inner nebulae seem also to share a similar axis. In particular, the Western and Northern arcs (see in Fig. 7) appear to converge to a point lying within or behind the Weigelt complex. This position is rather symmetrical to the position of the SE clump. The butterfly shape itself is suggested by two other protruding regions, namely the NE and the SW clumps (Fig. 7). Such a symmetry is potentially highly informative on the physical processes acting close to the star. Could it be that these structures are the sky projection of 3D optically thin geometry? Such an interpretation is at the moment premature. It should be of greatest interest to measure the radial velocity of the rims, but the combination of spectral and spatial resolution required is difficult to attain ${ }^{3}$.

A complex relationship must exist between the IR Butterfly nebula and the Little Homunculus, discovered by Ishibashi et al. (2003) with the HST, which is supposed also to be a consequence of the eruption of 1890 . This structure is seen in emission lines at visual wavelengths, while the IR images are dominated by dust emission which makes it difficult to compare the two geometries. However the similarity of their spatial extensions (about $2^{\prime \prime}$ ) probably points to a common origin of the structures. Ishibashi et al. (2003) showed that the polar caps of the Little Homunculus are expanding outward at about $300 \mathrm{~km} \mathrm{~s}^{-1}$. The present polar wind is much faster, of the order of $1000 \mathrm{~km} \mathrm{~s}^{-1}$ and it carries a high flux of mass (Smith et al. 2003b, see in particular their Fig. 7). Dwarkadas \& Owocki (2002) predict a mass-flux difference of a factor of about five in the polar and equatorial direction. At this speed the wind ejected about $40 \mathrm{yr}$ ago should have impacted this preexisting slow motion structure supposedly ejected in 1890 . Of course we assume that the latitudinal dependence detected in 2003 was already present by that time. We suspect that the conditions for dust formation could be phenomenologically equivalent to the ones encountered in the dusty $\mathrm{WR}+\mathrm{O}$ binary as proposed recently by Smith et al. (2004a). The rims of the butterfly nebula are probably places where strong density gradients are combined with high temperatures. The fast current wind of Eta Car may impact strongly upon these rims, providing the conditions for an efficient dust formation.

Any slow dense material in the vicinity of Eta Car (i.e. within $2^{\prime \prime}$ ) has to face three spatially localized regimes of wind. The polar regions of the inner nebula are facing a dense and fast wind, the intermediate-latitude regions experience fast but probably less dense wind, and the equatorial regions receive an equatorial wind with considerably less kinetic energy. Moreover there is a considerable shielding close the the equatorial plane in the direction of the Weigelt complex. It is well established that this zone (which contains the so-called "strontium region") presents fairly low excitation condition compatible with an efficient dust processing (Hartmann et al. 2004) but it is also relatively devoid of dust compared to other parts of the Homunculus nebula. From the previous considerations, we expect a latitudinal modulation of the survival probability of any dense dusty structure in the vicinity of Eta Car.

Finally, the consequences of the binarity of Eta Car are probably large and we now discuss some potential consequences of the wind-wind collision on the dust lying close to the equatorial plane.

\subsection{Effect of the binary orbit on the equatorial ejecta}

The Weigelt complex has been extensively discussed in many papers (Weigel \& Ebersberger 1986; Hoffman \& Weigelt 1988; Weigelt et al. 1995, 1996) and it is now well established that it

3 The NACO spectral resolution in spectroscopic mode being limited to $R \approx 1500$, i.e. $200 \mathrm{~km} \mathrm{~s}^{-1}$. 
lies at least close to the equatorial plane (Davidson et al. 1996; Smith et al. 2004a; Smith 2004). As stated previously, in the NACO images the Weigelt complex embeds the star and there is no apparent break between this structure and and so-called SE filament, which shares the same apparent axis of symmetry. In this section we tentatively attribute the shape and mass content of the SE filament to the effect of a wind-wind collision with a secondary star during and after the outbursts.

The SE filament could be the physical counterpart of the Weigelt complex, on the partly obscured receding part of the equatorial plane. This might explain the flux difference between the two regions although a differing illumination from the central star may also contribute. However, a more straightforward explanation is that the amount of material is simply much less than in the Weigelt complex. The absence of dust emission in the North-East must also be incorporated into a global interpretation.

The global effects of the 5.52 cycle on the X-ray (Corcoran et al. 2001), the optical (Damineli 1996; Damineli et al. 2000; Smith et al. 2000; van Genderen et al. 2001; Martin et al. 2004) and the NIR (Smith \& Gehrz 2000; Whitelock 2004) flux are now better understood, although the secondary characteristics and its orbital parameters are still unconstrained. Two facts appear unavoidable: the period of the orbit is rigorously established and the eccentricity of the system must be large.

If we place the orbit of the binary such that the periastron is located between the Weigelt complex and the star, and the apastron in the North-East, we can interpret the apparent equatorial structures by a competing effect between the winds and radiative fluxes of the primary and the secondary. When the secondary is at periastron, its cone wind, dominated by fast and diffuse gas, passes the Weigelt complex relatively rapidly, limiting the amount of dust destroyed at each passage and explaining the remanence of such a large amount of material close to the star. The binary model could also help explaining the formation of an equatorial ejection of large and dense clumps at low velocities during the outburst of 1890 . On the other hand, the dust material situated in the North-East could have been efficiently cleaned out during the 18 orbits since the 1890's outburst by the slower passage of the secondary wind cone. Finally, dust may still form in the equatorial plane refuelling the SE filament and the Weigelt complex at each orbit as seen in pinwheel nebulae (Tuthill et al. 1999). The dichotomy between the North-East empty region and the SE filament is easily explained in the context of a pinwheel nebula by the tilt angle of the cone of the secondary wind compared to the secondary orbital motion.

This hypothesis can be tested by a monitoring of the UV emission in this region (part of the "purple Haze"). Recently, Smith et al. (2004a) and Smith \& Morse (2004) presented such a monitoring of the emission from the "purple Haze" and proposed a model of binary orbit very similar to the one what has been discussed above. However, they place the periastron in the North-East of the primary star, while we are tempted to put it in the South-West in order to explain the large content and survivance of the Weigelt complex.

\subsection{Temporal variability}

Great care must be taken when placing the data presented here in the frame of the 5.52-year cycle of Eta Car. We are dealing with data of unprecedented spatial resolution and the variability effects of the individually resolved features such as the sublimation radius or the MIR emission of the Weigelt complex are largely unknown. With the VLT and techniques like Adaptive Optics and Long Baseline Interferometry the spatial resolution is such that the expected time lag between a central star event and its impact on our data is expected to be fairly short. Flow time is given approximately by:

$t=23.7(d / 2.5 \mathrm{kpc})\left(r / 1^{\prime \prime}\right)\left(500 \mathrm{~km} \mathrm{~s}^{-1} / v\right)(\mathrm{yr})$.

Thus for $d=2.3 \mathrm{kpc}$, and $r=0.1^{\prime \prime}$ the flow time is about 2.2 years. The effect of a rapid change of the UV and visual luminosity of the central object could affect the sublimation radius even faster.

Both NACO images and MIDI data must consequently be situated in the frame of the 5.52-year periodicity. Since accurate photometry of the NACO images is difficult we rely more on a monitoring of the geometry of Eta Car alone. Short time scale variability ( $\sim$ a month) can represent a real problem in interpreting MIDI data at different projected baselines separated by a large time lag. This question is of importance since it is difficult to observe a large number of baselines during a time interval smaller than the variability expected in mid-IR, which corresponds to the time needed to form dust and move it away.

The NACO images have been recorded in December 2002 at phase 0.87 , but the most of the MIDI data have been recorded in June 2003 at phase close to 1., just before the shell ejection event. Whitelock et al. (2003) reported in June 2003 that the anticipated fading of Eta Car at infrared wavelengths (JHKL) started between June 19 ( $L$-band) and June 24 ( $J$-band), i.e. just after the MIDI observations.

Large extinction effects are expected from shell ejection from the primary star, which may be periodically triggered by a companion. These shell ejections are implied by radio observations (Feast et al. 2001). These ejections are expected to offset the radius of dust sublimation, which should be a great indicator of dust formation and indirectly of the amount of ionizing photons that can reach the different parts of the nebula. Smith et al. (2000) have clearly demonstrated that the variability detected by HST can be attributed either to a bolometric variation of the star or, more probably, to grain destruction, which could then explain a decrease in circumstellar extinction. A shift of the dust forming regions may also affect the extinction.

Further observations with the same settings should provide more information on the real performances of a monitoring of Eta Car by the instruments NACO and MIDI.

\section{Conclusion}

We have reported observations of Eta Car by NACO and MIDI. We can draw the following conclusions:

- We have obtained near- and mid-IR images of the inner few arcsec of the core of Eta Car, using the VLT AO system 
NACO and the VLTI/MIDI interferometer, at an (unprecedented) spatial resolution of 60 and 140 milliarcsec respectively. The central 3 arcsec region of the dusty nebula has been observed in great detail.

- The Weigelt complex of "blobs" is seen in detail for the first time in the thermal infrared, and their position can be measured accurately. The IR counterparts of optical clumps C and $\mathrm{D}$ can be identified, whereas the clump $\mathrm{B}$ is not detected. These visible structures, dominated by scattering, may trace the walls of the dust clumps seen in the IR. A fainter structure, the SE filament may be the southern counterpart of the Weigelt complex.

- The central object is seen by NACO to be separated from its surroundings. A large area empty of dust of a typical radius of $0 .{ }^{\prime} 10-0 . ' 15$ (230-350 AU) is detected.

- We have detected a chemical difference between the dust located at the Weigelt complex and that located at the south-east of the star. Aluminium oxide (corundum) seems to dominate the spectrum of the SE clump. This molecule is one of the first dust species to condense in a harsh environment.

- These Adaptive Optic and Interferometric observations allow the extraction of upper limits to the SED of the central object from $3.7 \mu \mathrm{m}$ to $13.5 \mu \mathrm{m}$.

- We have discussed on the spatial geometry of the dusty nebula. In particular, we have outlined that the bright dusty rims seem to share the axis of symmetry of the Homunculus.

- We hypothesize that the survival and the large mass of the Weigelt blobs could be related to their location close to the periastron of the system orbit, where the secondary star is strongly extinguished by the primary dense wind. The counterpart of this high density region is an almost empty one in the North-East and the faint SE filament which could be the consequence of the eccentric orbital motion of the secondary.

Acknowledgements. Working on developing new techniques and new instruments is still a great challenge which is always risky and not always rewarding. The authors warmly thank the technical people of the MIDI team and the ESO VLTI team working on Paranal observatory or in Garching. They made possible the advent of MIDI whose performances can not be dissociated from the ones of the impressive interferometric infrastructure of the VLTI.

\section{References}

Abraham, Z., Dominici, T. P., Damineli, A., et al. 2003, IAUC, 8162, 4

van Boekel, R., Kervella, P., Schöller, M., et al. 2003, A\&A, 410, L37

Chesneau, O., Verhoelst, T., Waters, L. B. F. M., et al. 2004, A\&A, submitted

Cohen, M. 1998, AJ, 115, 2092

Corcoran, M. F. 2003, IAUC, 8160, 3
Corcoran, M. F., Ishibashi, K., Swank, J. H., \& Petre, R. 2001, ApJ, 547,1034

Damineli, A. 1996, ApJ, 460, L49

Damineli, A., Kaufer, A., Wolf, B., et al. 2000, ApJ, 528, L101

Davidson, K. 1999, ASP Conf. Ser., 179, 304

Davidson, K., \& Humphreys, R. M. 1997, ARA\&A, 35, 1

Davidson, K., Dufour, R. J., Walborn, N. R., \& Gull, T. R. 1986, ApJ, 305,867

Davidson, K., Ebbets, D., \& Morse, J. A. 1997, AJ, 113, 335

Davidson, K., Ishibashi, K., Gull, T. R., et al. 2000, ApJ, 530, L107

Davidson, K., Smith, N., Gull, T. R., et al. 2001, AJ, 121, 1569

Dominik, C., Dullemond, C. P., Cami, J., \& van Winckel, H. 2003, A\&A, 398, 607

Dorland, B. N., Currie, D. G., \& Hajian, A. R. 2004, AJ, 127, 1052

Dorschner, J., Begemann, B., Henning, T., et al. 1995, A\&A, 300, 503

Draine, B. T., \& Lee, H. M. 1984, ApJ, 285, 89

Dumm, T., \& Schild, H. 1998, NewA, 3, 137

Duncan, R. A., \& White, S. M. 2003, MNRAS, 338, 425

Duncan, R. A., White, S. M., Reynolds, J. E., \& Lim, J. 1999, ASP Conf. Ser., 179, 54

Dwarkadas, V. V., \& Owocki, S. P. 2002, ApJ, 581, 1337

Falcke, H., Davidson, K., Hofmann, K.-H., \& Weigelt, G. 1996, A\&A, 306, L17

Feast, M., Whitelock, P., \& Marang, F. 2001, MNRAS, 322, 2001

Fluks, M. A., Plez, B., The, P. S., et al. 1994, A\&AS, 105, 311

van Genderen, A. M., de Groot, M., \& Sterken, C. 2001, ASP Conf. Ser., 233, 59

van Genderen, A. M., \& Sterken, C. 2004, A\&A, 423, L1

Hartmann, H., Gull, T., Johannson, S., et al. 2004, A\&A, 419, 215

Hillier, D. J., \& Allen, D. A. 1992, A\&A, 262, 153

Hillier, D. J., Davidson, K., Ishibashi, K., \& Gull, T. 2001, ApJ, 553, 837

Hofmann, K.-H., \& Weigelt, G. 1988, A\&A, 203, L21

Humphreys, R. M. 2003, IAUS, 212, 38

Ishibashi, K., Gull, T. R., Davidson, K., et al. 2003, ApJ, 125, 3222

Lamers, H. J. G. L. M., Livio, M., Panagia, N., \& Walborn, N. R. 1998, ApJ, 505, L131

Leinert, Ch., Graser, U., Waters, L. B. F. M., et al. 2003a, SPIE, 4838, 893

Leinert, Ch., Graser, U., \& Przygodda, F. 2003b, Ap\&SS, 286, 73

Leinert, C., van Boekel, R., \& Waters, L. B. F. M. 2004, A\&A, 423, 537

Lenzen, R., Hartung, M., Brandner, W., et al. 2003, SPIE, 4841, 944

Maldoni, M. M., Egan, M. P., Robinson, G., et al. 2004, MNRAS, 349, 665

Martin, J. C., Koppelman, D., the HST Eta Car Treasury Project Team 2004, AJ, 127, 2352

Mitchell, R. M., \& Robinson, G. 1978, ApJ, 220, 841

Mitchell, R. M., \& Robinson, G. 1986, MNRAS, 222, 347

Morris, P. W., Waters, L. B. F. M., Barlow, M. J., et al. 1999, Nature, 402, 502

Morse, J. A., Davidson, K., Bally, J., et al. 1998, AJ, 116, 2443

Morse, J. A., Kellogg, J. R., Bally, J., et al. 2001, ApJ, 548, L207

Nota, A., Livio, M., Clampin, M., \& Schulte-Ladbeck, R. 1995, ApJ, 448,788

Pantin, E., \& Le Mignant, D. 2000, A\&A, 355, 155

Petrov, R. G., Malbet, F., Weigelt, G., et al. 2003, SPIE, 4838, 924

Pittard, J. M., \& Corcoran, M. F. 2002, A\&A, 383, 636

Przygodda, F., Chesneau, O., Graser, U., et al. 2003, Ap\&SS, 286, 85

Rigaut, F., \& Gehring, G. 1995, Rev. Mex. Astron. Astrofis., 2, 27 
Robinson, G., Mitchell, R. M., Aitken, D. K., et al. 1987, MNRAS, Tielens, A. G. G. M. 1990, in ed. M. O. Menessier, \& A. Omont, 227,535

Rousset, G., Lacombe, F., Puget, P., et al. 2003, SPIE, 4839, 140

Smith, N. 2002, MNRAS, 337, 1252

Smith, N. 2004, AJ, 127, 2793

Smith, N., \& Gehrz, R. D. 2000, ApJ, 529, L99

Smith, N., \& Morse, J. A. 2004, ApJ, 605, 854

Smith, N., Morse, J. A., Davidson, K., \& Humphreys, R. M. 2000, AJ, 120,920

Smith, N., Gehrz, R. D., Hinz, P. M., et al. 2002, ApJ, 567, L77

Smith, N., Gehrz, R. D., Hinz, P. M., et al. 2003a, AJ, 125, 1458

Smith, N., Davidson, K., Gull, T. R., et al. 2003b, ApJ, 586,432

Smith, N., Morse, J. A., Gull, T. R., et al. 2004a, ApJ, 605, 405

Smith, N., Morse, J. A., Collins, N. R., \& Gull, T. R. 2004b, ApJ, 610, L105 Editions Frontières, Gif-sur-Yvette, 186

Thuthill, P. G., Monnier, J. D., \& Danchi, W. C. 1999, Nature, 398, 487

Waters, L. B. F. M., Morris, P. W., Voors, R. H. M., \& Lamers, H. J. G. L. M. 1997, ASP Conf. Ser., 120, 326

Weigelt, G., \& Ebersberger, J. 1986, A\&A, 163, L5

Weigelt, G., Albrecht, R., Barbieri, C., et al. 1995, Rev. Mex. Astron. Astrofis., Ser. Conf., 2, 11

Weigelt, G., Davidson, K., \& Hofmann, K.-H. 1996, A\&A, 306, L17

Whitelock, P. A., Marang, F., \& Crause, L. 2003, IAUC, 8160, 2

Whitelock, P. A., Feast, M. W., Marang, F., \& Breedt 2004, MNRAS, accepted

Westphal, J. A., \& Neugebauer, G. 1969, ApJ, 156, L45

Wright, A. E., \& Barlow, M. J. 1975, MNRAS, 170, 41 